\title{
Excess iron modulates endoplasmic reticulum stress- associated pathways in a mouse model of alcohol and high-fat diet-induced liver injury
}

\author{
Terrence CH Tan ${ }^{1,2,3}$, Darrell HG Crawford ${ }^{1,2}$, Lesley A Jaskowski ${ }^{1,2}, V_{\text {Nathan Subramaniam }}^{1,2,4}$, \\ Andrew D Clouston ${ }^{1}$, Denis I Crane ${ }^{5}$, Kim R Bridle ${ }^{1,2}$, Gregory J Anderson ${ }^{4}$ and Linda M Fletcher ${ }^{1,3}$
}

Endoplasmic reticulum (ER) stress is an important pathogenic mechanism for alcoholic (ALD) and nonalcoholic fatty liver disease (NAFLD). Iron overload is an important cofactor for liver injury in ALD and NAFLD, but its role in ER stress and associated stress signaling pathways is unclear. To investigate this, we developed a murine model of combined liver injury by co-feeding the mildly iron overloaded, the hemochromatosis gene-null ( $\mathrm{Hfe}^{-}$) mouse ad libitum with ethanol and a high-fat diet (HFD) for 8 weeks. This co-feeding led to profound steatohepatitis, significant fibrosis, and increased apoptosis in the $\mathrm{Hfe}^{-/-}$mice as compared with wild-type (WT) controls. Iron overload also led to induction of unfolded protein response (XBP1 splicing, activation of IRE-1 $\alpha$ and PERK, as well as sequestration of GRP78) and ER stress (increased CHOP protein expression) following HFD and ethanol. This is associated with a muted autophagic response including reduced LC3-I expression and impaired conjugation to LC3-II, reduced beclin-1 protein, and failure of induction of autophagy-related proteins (Atg) 3,5, 7, and 12. As a result of the impaired autophagy, levels of the sequestosome protein p62 were most elevated in the $\mathrm{Hfe}^{-/-}$group co-fed ethanol and HFD. Iron overload reduces the activation of adenosine monophosphate protein kinase associated with ethanol and HFD feeding. We conclude that iron toxicity may modulate hepatic stress signaling pathways by impairing adaptive cellular compensatory mechanisms in alcohol- and obesity-induced liver injury.

Laboratory Investigation (2013) 93, 1295-1312; doi:10.1038/labinvest.2013.121; published online 14 October 2013

KEYWORDS: autophagy; endoplasmic reticulum stress; HFE; iron overload; unfolded protein response

Iron is an important cofactor in the progression of chronic liver injury caused by alcoholic (ALD) and nonalcoholic fatty liver disease (NAFLD). Excessive alcohol consumption and risk factors for NAFLD (obesity, hypertension, and the metabolic syndrome) often coexist and share many similar pathophysiological characteristics. ${ }^{1}$ Increased iron indices are frequently reported in ALD and NAFLD, where iron is regarded as an important determinant of progressive liver injury. ${ }^{2-4}$ Patients with the genetic hemochromatosis who consume excessive amounts of alcohol have greatly increased risk of cirrhosis, and NAFLD patients with increased hepatic iron have greater levels of fibrosis than those without. ${ }^{5}$

The pathogenesis by which iron promotes further liver injury in ALD and NAFLD has been relatively unclear. Its redox potential makes iron an effective agent in generating reactive oxygen species (ROS). ${ }^{6}$ This may lead to tissue oxidative stress, lipid peroxidation of cellular membranes, fibrosis, and damage to subcellular organelles such as the lysosomes and mitochondria. ${ }^{6}$ These mechanisms share similarities with those of ALD and NAFLD, where oxidative stress may occur concomitantly. Previous studies have shown that ironoverloaded rats fed ethanol may develop cirrhosis associated with marked lipid peroxidation and activation of multiple profibrogenic genes. ${ }^{7}$ Hepatic iron overload has also been shown to alter glucose homeostasis and lipid metabolism in promoting diet-induced hepatic steatosis in animal models. ${ }^{8}$ Iron accumulation may also have a role in activating Kupffer cells to release proinflammatory cytokines. ${ }^{9}$ However, the

\footnotetext{
${ }^{1}$ School of Medicine, University of Queensland, Brisbane, QLD, Australia; ${ }^{2}$ Gallipoli Medical Research Centre, Greenslopes Hospital, Brisbane, QLD, Australia; ${ }^{3}$ Department of Gastroenterology and Hepatology, Princess Alexandra Hospital, Brisbane, QLD, Australia; ${ }^{4}$ Queensland Institute of Medical Research, Brisbane, QLD, Australia and ${ }^{5}$ Eskitis Institute for Cell and Molecular Therapies and School of Biomolecular and Physical Sciences, Griffith University, Brisbane, QLD, Australia Correspondence: Dr TCH Tan, MBBS, FRACP, PhD, Department of Gastroenterology and Hepatology, Princess Alexandra Hospital, Ipswich Road, Brisbane, QLD 4102, Australia.

E-mail: perthfracp@gmail.com

Received 26 June 2013; revised 16 September 2013; accepted 18 September 2013
} 
mechanisms by which iron promotes fibrosis in ALD and NAFLD needs further elucidation.

Stress signaling pathways refer to a diverse group of cellular responses to exogenous and endogenous sources of metabolic and biochemical insults. Together, they preserve cell growth, differentiation, survival, as well as regulate cell death. A balance between prosurvival and proapoptotic pathways is crucial in health and disease states. The unfolded protein response (UPR) and autophagy are cellular stressadaptive response mechanisms that restore metabolic and energy homeostasis. The UPR mechanism is a chain of cellular events that responds to the accumulation of unfolded or misfolded proteins in the endoplasmic reticulum (ER). ${ }^{10}$ These pathways involve global attenuation of protein translation, increase in chaperone proteins, and ER expansion to increase protein folding as well as activation of the ER-associated degradation machinery to restore balance in the ER. ${ }^{10}$ Impaired UPR can lead to ER stress and prolonged ER stress may result in inflammation and apoptosis as implicated in ALD and NAFLD. ${ }^{11,12-14}$ The downstream effects of ER stress include activation of autophagy, c-Jun $\mathrm{N}$-terminal kinase (JNK) pathways, modulation of Toll-like receptor (TLR) signaling, and macrophage activation in alcohol- and obesity-induced synergistic liver injury. ${ }^{15-17}$ Autophagy, on the other hand, is the basic catabolic mechanism that involves cell degradation of unnecessary or dysfunctional cellular components through the lysosomal machinery. Defects in the autophagy machinery have also been implicated in the development of steatosis and ALD and NAFLD. ${ }^{18}$ However, the links between iron toxicity and modulation of the UPR/ER stress and autophagy in ALD and NAFLD remain unclear.

In this study, our aim was to create a murine model of experimental liver injury caused by concomitant iron excess and alcohol- and diet-induced obesity to investigate how iron may exacerbate combined ALD and NAFLD. We used a genetic model of hemochromatosis, the hemochromatosis gene-null $\left(\mathrm{Hfe}^{-{ }^{-}}\right)$mouse to represent mild hepatic iron overload and provided free access to ethanol and a high-fat diet (the ethanol and HFD co-feeding model). The development of advanced fibrosing steatohepatitis in these mice allowed us to determine the mechanistic interactions involving these co-toxins. Here we report that increased hepatic iron, even when mild to moderate, may synergistically exacerbate the hepatotoxic effects of combined alcohol- and diet-induced obesity. This is a large-scale study that attempts to identify multiple signaling pathways in which iron may interact with alcohol and HFD, in isolation or in combination, to promote liver injury, in the hope that specific common pathogenic mechanisms can be targeted for potential future therapies.

\section{MATERIALS AND METHODS}

\section{Experimental Protocols and Animal Models}

The animal protocol was approved by the Queensland Institute of Medical Research Animal Ethics Committee.
Six-week-old male wild-type (WT) (Animal Resources Centre, Western Australia) and hemochromatosis gene-null $\left(\mathrm{Hfe}^{-/-}\right)$mice on a C57/BL6 background (originally supplied by Professor William Sly, St Louis University, MO, USA, on a mixed background, but back-crossed for 10 generations; 6 weeks old) were housed in a temperature-controlled environment $\left(23^{\circ} \mathrm{C}\right)$, with a 12 -h light/dark cycle and allowed ad libitum access to diets and drinking water.

The animal protocol is illustrated in Supplementary Figure S1. For each genotype, the mice ( $n=6$-13/group) were randomly assigned to standard laboratory chow diet (control diet) and a high-fat, high-calorie diet (HFD, Specialty Feeds, Glen Forrest, WA, Australia), with or without ethanol added to the drinking water. The HFD had a 50\% higher caloric count than chow fat composition of the HFD by weight was $15 \%$ saturated fat, $6 \%$ polyunsaturated, and $2 \%$ monounsaturated fat, derived from a predominant mixture of cocoa butter $(50 \mathrm{~g} / \mathrm{kg})$, partially hydrogenated vegetable oil $(131 \mathrm{~g} / \mathrm{kg})$, and canola oil $(50 \mathrm{~g} / \mathrm{kg})$, with added cholesterol $(0.19 \%)$. Details of nutrient components of each diet are provided in Supplementary Figure S2. The concentration of ethanol was increased incrementally by $5 \%$ every 2 days over a 1-week period to reach a final concentration of $20 \%$ (vol/ vol). The mice received humane care according to the institution guidelines and were killed following 8 weeks of dietary treatment. Blood and liver samples were retrieved and stored at $-80^{\circ} \mathrm{C}$ before experiments described below.

\section{Serum Biochemistry}

Sera collected from blood during anesthesia and animal killing were measured for ethanol levels using the colorimetric Ethanol Assay Kit (Biovision, San Francisco, CA, USA). Serum alanine aminotransferase (ALT), iron, cholesterol, and triglyceride measurements were performed as previously described. ${ }^{19}$

\section{Quantitative Real-Time PCR Analysis}

Total cellular RNA was isolated from hepatic tissue using the TRIzol reagent (Invitrogen, Melbourne, Australia) according to the manufacturer's instructions. Reverse transcription and quantitative RT-PCR (Applied Biosystems, Brisbane, Australia) were performed as previously described. ${ }^{19}$ The primer sequences used for the experiments are outlined in Supplementary Table S3. Gene expression was normalized to the internal standard basic transcription factor 3 (Btf3).

\section{Histopathological Analysis}

Small pieces of liver tissue were fixed immediately in $10 \%$ buffered formalin phosphate and 3\% paraformaldehyde (Sigma). After paraffin embedding, $5-\mu \mathrm{m}$ transverse sections were prepared and stained with hematoxylin and eosin (H\&E). Hepatic fibrosis was assessed by Sirius Red staining. Presence of hepatic stellate cells was assessed by positive staining for $\alpha$-smooth muscle actin ( $\alpha$-SMA) (Sigma-Aldrich, St Louis, MO, USA) in hepatic sinuosids using immunohistochemistry (M.O.M Kit, Vector Laboratories, 
Burlingame, CA, USA). Formalin-fixed liver sections were processed for H\&E staining and evaluated for steatosis, hepatocyte ballooning, inflammatory cell infiltrates, and Mallory bodies. Steatosis, lobular inflammation, ballooning, and fibrosis were graded according to currently accepted criteria. ${ }^{20}$ Steatosis was classified as mild $(0-33 \%)$, moderate $(34-66 \%)$, or severe $(>66 \%)$. Hepatic lipid content was also semiquantitatively assessed using the Oil Red O staining on frozen liver sections.

\section{Hepatic Triglyceride Content}

Liver tissue $(50-100 \mathrm{mg})$ was homogenized in $1.15 \%$ potassium chloride. Triacylglycerol extraction was performed as previously described. ${ }^{21}$ Triglyceride content was assayed using a commercial kit (Wako Diagnostics, Richmond, VA, USA).

\section{Western Blots}

Protein extraction and western blot analysis were performed as previously described. ${ }^{19}$ Immunoblotting was performed with specific primary antibodies (Supplementary Table S4). Blocking and antibody dilutions were performed in $10 \%$ skim milk in Tris-buffered saline with $0.1 \%$ Tween. Protein quantification was performed by densitometry analysis using ImageJ (NIH, USA).

\section{Lipid Peroxidation and Antioxidant Status}

Hepatic lipid peroxidation was assessed by measuring the aldehydic by-products that are reactive with thiobarbituric acid (TBARS) as previously described. ${ }^{22}$ Desferrioxamine $(1 \mathrm{mM})$ was included in the reaction mix to chelate excess iron released as a consequence of the homogenization procedure. Total cellular reduced (GSH) and oxidized glutathione (GSSG) contents were measured on homogenized liver tissue using specific assay kits (Cayman Chemical Company, Ann Arbor, MI, USA).

\section{Mitochondrial Function Studies}

Mitochondrial function was assessed using the respiratory Complex 1 (NADH/ubiquinone oxidoreductase) and manganese superoxide dismutase (MnSOD) activities as previously described. ${ }^{19,23}$ Complex 1 activity was measured as described but at $30{ }^{\circ} \mathrm{C}$ and normalized to citrate synthase activity. ${ }^{23}$

\section{Hepatic Iron Content}

Hepatic non-heme iron concentration (HIC) was measured as previously described. ${ }^{24}$ Semiquantitative grading of hepatic iron was also performed on liver sections following Perls' Prussian Blue staining as previously described. ${ }^{25}$

\section{Assessment of Hepatocellular Apoptosis}

Assessment of hepatocellular death by the apoptotic pathway was performed using the terminal deoxynucleotidyl transferase (TdT) dUTP Nick-End Labeling (TUNEL) assay according to the manufacturer's instructions (Apoptag
Peroxidase In Situ Apoposis Detection Kit, Merck Millipore, Billerica, MA, USA).

\section{Statistical Analysis}

Statistical analysis was performed using the GraphPad Prism 5 Software (GraphPad, San Diego, CA, USA). Results are expressed as the mean \pm s.e.m. Comparisons between two groups were made using Student's $t$-test. A two-way analysis of variance (ANOVA) was used to compare the means of multiple groups to assess the effects of diet and ethanol, followed by Bonferroni post hoc test between groups. A $P$-value of $<0.05$ was used to define statistical significance.

\section{RESULTS}

\section{Body Weights and Serum Indices}

Baseline body weights were not significantly different across all mouse groups (data not shown). Monitoring of food intake and drinking did not reveal any significant differences between genotypes and ethanol or nonethanol groups (data not shown). The mean body weight gain of mice fed the HFD for 8 weeks either alone or in combination with ethanol was significantly increased when compared with chow-control feeding, regardless of genotype, but was particularly high for $\mathrm{Hfe}^{-{ }^{-}}$mice on the HFD (Table 1). However, ethanol consumption attenuated the weight gain caused by the HFD for each genotype. Notably, despite the similar amounts of ethanol consumption ( $10 \mathrm{ml} /$ animal/week), $\mathrm{Hfe}^{-/-}$mice achieved a near 2.5 -fold elevation in serum ethanol concentrations compared with their WT counterparts on comparable diets (Table 1).

Serum iron concentrations were significantly higher in the $\mathrm{Hfe}^{-1-}$ animals, and were increased even further with ethanol feeding (Table 1). Serum triglyceride and cholesterol were significantly lower in $\mathrm{Hfe}^{-/-}$mice compared with WT controls, but increased with HFD (with or without ethanol) feeding to values similar to the WT on similar diets.

\section{Effects on Hepatic Iron Content}

HIC was significantly higher throughout the $\mathrm{Hfe}^{-/-}$groups as compared with the WT animals (Figure 1a). However, a HFD feeding resulted in a decreased HIC, although this effect was not seen in those mice that received ethanol treatment. On average, the HIC was twofold higher in $\mathrm{Hfe}^{-/-}$compared with WT mice. Perls' staining showed an iron staining pattern that is predominantly hepatocellular and periportal in location in the $\mathrm{Hfe}^{-1-}$ mice, but those fed chow or HFD with ethanol also displayed some reticuloendothelial iron deposition (Figure 1b). Iron staining was not evident in any of the WT mice.

\section{Iron Overload Augments Steatohepatitis and Liver Injury Caused by Ethanol and HFD}

Steatosis was noted with HFD alone and in all groups that received the HFD (Figure 2a). The degree of steatosis was further exacerbated when HFD was combined with ethanol 
Table 1 Comparison of body weights and serum parameters

\begin{tabular}{|c|c|c|c|c|c|c|c|c|}
\hline \multirow[b]{2}{*}{ Parameters } & \multicolumn{4}{|c|}{ WT } & \multicolumn{4}{|c|}{$H f e^{-/-}$} \\
\hline & Chow & Chow $+E$ & HFD & $\mathrm{HFD}+\mathrm{E}$ & Chow & Chow $+E$ & HFD & $\mathrm{HFD}+\mathrm{E}$ \\
\hline \multicolumn{9}{|l|}{ Weight } \\
\hline$\Delta$ Total body (g) & $4.3 \pm 0.7$ & $4.5 \pm 0.6$ & $12.8 \pm 1.0^{\mathrm{a}}$ & $10.5 \pm 1.7^{\mathrm{a}}$ & $4.8 \pm 0.6$ & $4.3 \pm 1.5$ & $19.1 \pm 0.7^{\mathrm{a}}$ & $8.5 \pm 1.7^{\mathrm{a}}$ \\
\hline Liver/body (\%) & $4.52 \pm 0.06$ & $5.21 \pm 0.06$ & $4.12 \pm 0.14$ & $4.71 \pm 0.13$ & $4.63 \pm 0.10$ & $4.02 \pm 0.42$ & $5.45 \pm 0.40^{\mathrm{a}}$ & $4.76 \pm 0.29$ \\
\hline \multicolumn{9}{|l|}{ Serum $(\mathrm{mmol} / \mathrm{l})$} \\
\hline Ethanol (mM) & ND & 11.9. \pm 0.5 & ND & $12.6 \pm 0.8$ & ND & $27.7 \pm 1.2^{\mathrm{a}}$ & ND & $30.2 \pm 1.1^{\mathrm{a}}$ \\
\hline Iron $(\mu \mathrm{mol} / \mathrm{l})$ & $23.1 \pm 1.4$ & $24.3 \pm 1.2$ & $22.2 \pm 0.8$ & $24.1 \pm 1.1$ & $32.2 \pm 0.8^{\mathrm{a}}$ & $33.2 \pm 0.7^{\mathrm{a}}$ & $34.0 \pm 0.90^{\mathrm{a}}$ & $43.4 \pm 1.2^{\mathrm{a}}$ \\
\hline
\end{tabular}

Abbreviations: HFD, high-fat diet; $\mathrm{E}$, ethanol; ND, not detected; VAT, visceral adipose tissue; $\Delta=$ gain.

Values expressed as mean \pm s.e.m.

${ }^{\mathrm{a}} P<0.05$ compared with WT mice on chow diet only.

${ }^{\mathrm{b}} P<0.05$ compared with nonethanol animals within the same genotype and dietary group.

or with increased hepatic iron (Figure 2a). The combination of HFD, ethanol, and iron does not appear to increase hepatic steatosis any further compared with the iron-overloaded animals that received either HFD alone or ethanol alone (Figure 2a). Similarly, with regard to hepatocellular inflammation, a HFD or ethanol alone did not lead to any significant inflammation in the WT groups. Ethanol in combination with a HFD led to a mild degree of inflammatory injury, as assessed by lobular and portal inflammatory scores (Figures $2 \mathrm{~b}$ and $\mathrm{c}$ ) as well as serum ALT (Figure 2d). Ethanol and HFD co-feeding led to a threefold increase in serum ALT in the WT animals, but ALT was unaffected by either ethanol or HFD alone (Figure 2d). In isolation, the increased iron did not lead to any degree of liver injury or inflammation. However, the increased hepatic iron led to marked steatohepatitis in the $\mathrm{Hfe}^{-/-}$mice that were fed ethanol, or HFD alone, or in combination (Figures $2 \mathrm{~b}-\mathrm{d}$ ). The rises in serum ALT were most marked in the iron and HFD group, with a sevenfold rise following HFD as compared with WT or $\mathrm{Hfe}^{-/-}$groups fed chow diet alone. Interestingly, $\mathrm{Hfe}^{-/-}$mice on chow and ethanol also developed significant elevations in ALT with histologically similar degrees of liver inflammation as the HFD-fed animals (Figures 2a and d). The addition of ethanol to HFD and iron did not lead to further increases in hepatocellular inflammation or injury.

In the WT animals, compared with the chow-only group, there was a 3.5-fold increase in hepatic triglyceride content associated with HFD feeding, 2.5-fold with ethanol and chow, and fivefold with combined HFD and ethanol co-feeding (Figure 3a). In contrast, HFD feeding led to a 10fold rise in hepatic triglyceride levels over nonethanol chow controls in the $\mathrm{Hfe}^{-{ }^{-}}$animals (Figure $3 \mathrm{a}$ ), but the addition of ethanol to HFD did not lead to further accumulation of hepatic triglycerides. The biochemical quantification of hepatic triglyceride levels is further confirmed by findings on the Oil Red O staining, with maximal staining intensity in the groups with iron overload and HFD (Figure 3b).

\section{Iron Overload Exacerbates HFD- and Ethanol-Induced Hepatic Fibrosis}

On Sirius Red staining, neither HFD nor ethanol, either alone or in combination, caused any development in hepatocellular fibrosis in the WT mice. In the $\mathrm{Hfe}^{-1-}$ mice, the presence of increased iron alone did not cause any fibrosis either. However, the presence of increased iron, when combined with HFD or ethanol alone, led to significant subsinusoidal fibrosis, as evident in the $\mathrm{Hfe}^{-1-}$ mice (Figure 4a). $\mathrm{Hfe}^{-I_{-}}$ mice on chow and ethanol developed more extensive pericellular and subsinusoidal fibrosis than those on HFD alone. Portal-portal tract linkage fibrosis was seen in mice with increased iron and ethanol with or without HFD (Figure 4a). Consistent with the observed fibrosis, expression profiles for fibrogenic genes (Colla1, Mmp-2, and Timp-1, Figure 4b) were highest in these groups of animals. Evidence of hepatic stellate cell activation was demonstrated by the presence of positive staining $\alpha$-SMA on immunohistochemistry (Figure 5a) as well as elevated mRNA expression on PCR (Figure 5b). Consistent with increased hepatic stellate cell activation, the gene expression for transforming growth 


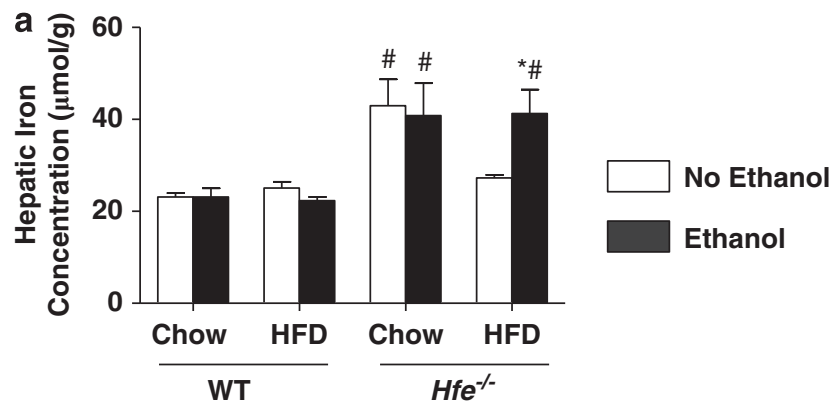

b
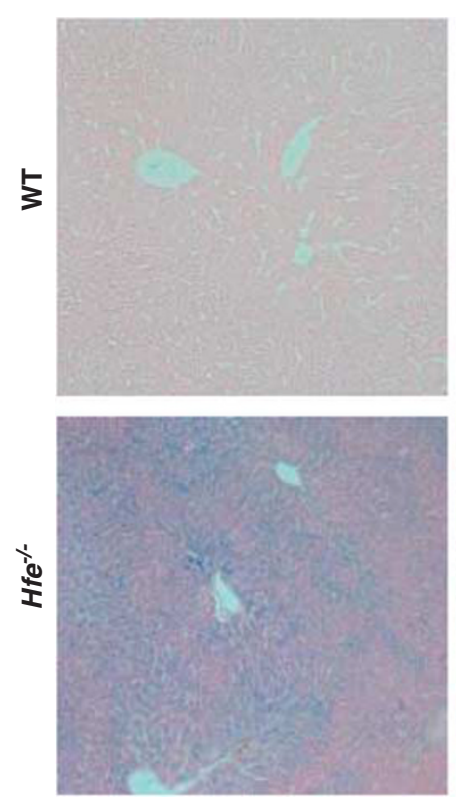

-
Chow
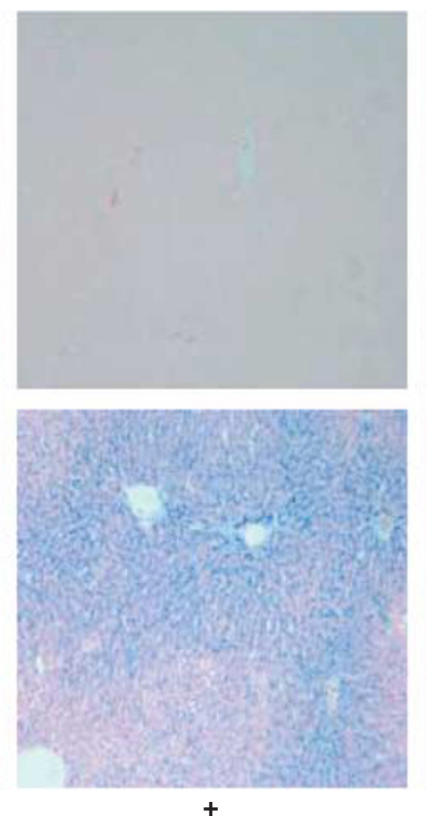

HFD
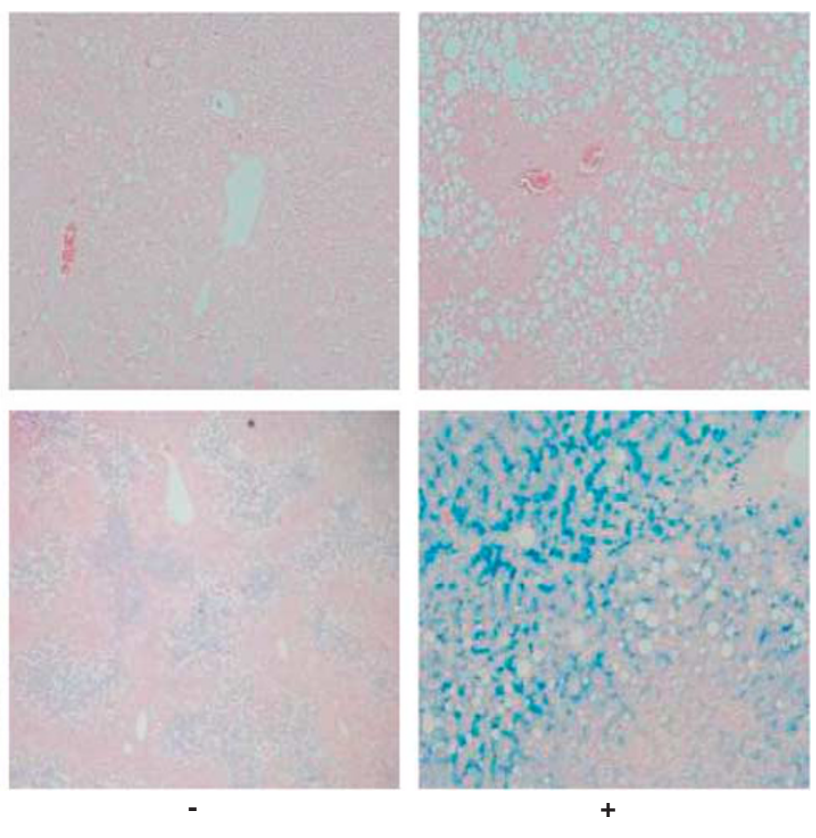

ETHANOL

Figure 1 Assessment of hepatic iron. (a) Hepatic iron concentrations and (b) Perls' staining for iron deposition in liver sections ( $\times 200$ magnification). ${ }^{\#} P<0.05$ compared with WT mice on chow only. ${ }^{*} P<0.05$ compared with nonethanol-treated animals within the same genotype and dietary group.

factor- $\beta 1($ Tgf- $\beta 1)$ revealed the greatest upregulation in $\mathrm{Hfe}^{-/-}$animals fed ethanol and HFD (Figure 5b).

\section{Iron Affects Hepatic Lipid Handling and Adiponectin Response to Ethanol and HFD}

Given that the degree of steatosis was most severe in the ironloaded animals fed HFD with or without ethanol, we investigated the effects of iron on hepatic lipid metabolism by measuring gene expression and protein levels of key lipid transcription factors. The mRNA expression of the key hepatic lipogenic transcription factor sterol regulatory element binding protein-1c (Srebp-1c) was increased with HFD or ethanol alone in the WT groups, but was highest with iron overload and HFD, with or without ethanol (Figure 6a). However, surprisingly, a HFD did not result in increased expression of SREBP downstream target genes fatty acid synthase (Fasn) or stearoyl CoA desaturase-1 (Scd1)
(Supplementary Figure S5). Ethanol consumption significantly increased Fasn and $S c d 1$ expression but only in the WT groups on chow and not in any of the $\mathrm{Hfe}^{-1-}$ animals. Similarly, increased hepatic iron significantly increased ethanol- and HFD-induced mRNA expression of the lipid trafficking and chaperone protein liver fatty acid binding protein (Lfabp) (Figure 6b), although these changes were not consistently seen with the other regulators of fatty acid entry (Cd36) (Figure 6c) and storage (peroxisome proliferatoractivated receptor- $\gamma$ (Ppar- $\gamma$ ); Figure $6 \mathrm{~d})$.

A HFD resulted in a 2.5 -fold increase in the mRNA expression of fatty acid $\beta$-oxidation gene Ppar- $\alpha$ in the WT animals, but this increase was abrogated with ethanol and/or iron overload, with the $\mathrm{Hfe}^{-/-}$failing to upregulate this gene following HFD or ethanol or both (Figure 6e). Either ethanol or HFD feeding led to increased hepatic expression of adiponectin receptor-2 (AdipoR2) in the WT mice, which 
a
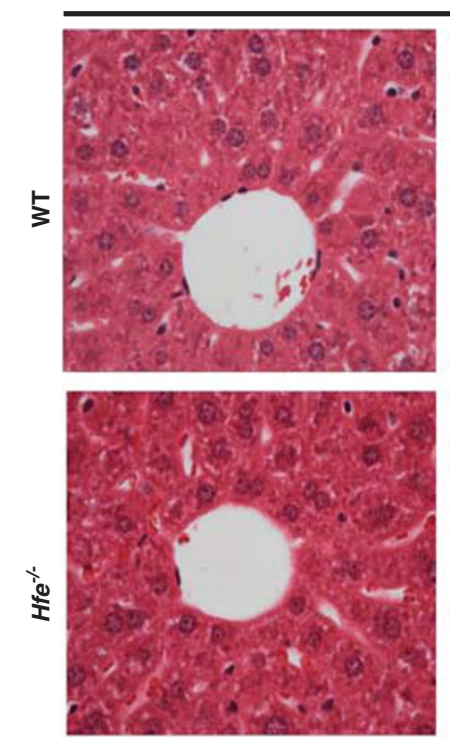

Chow
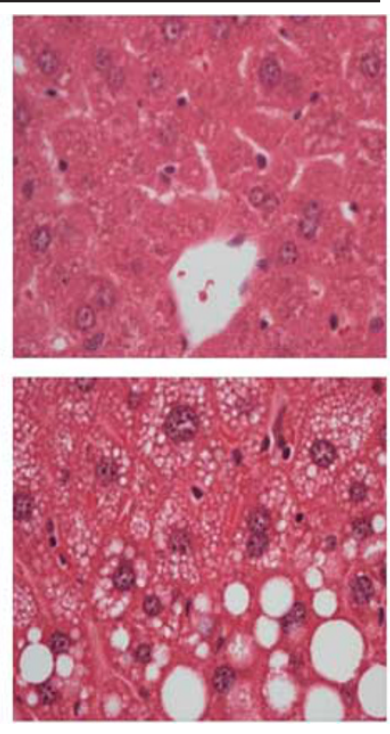

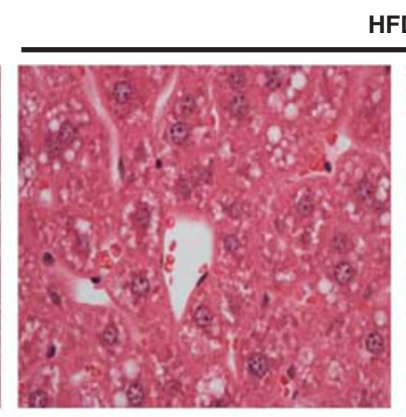

HFD

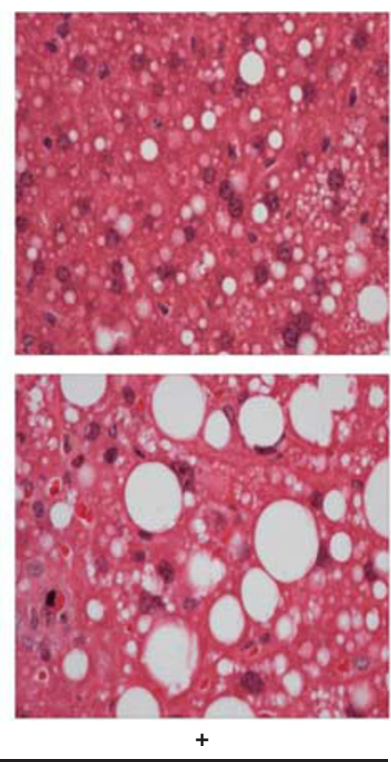

ETHANOL
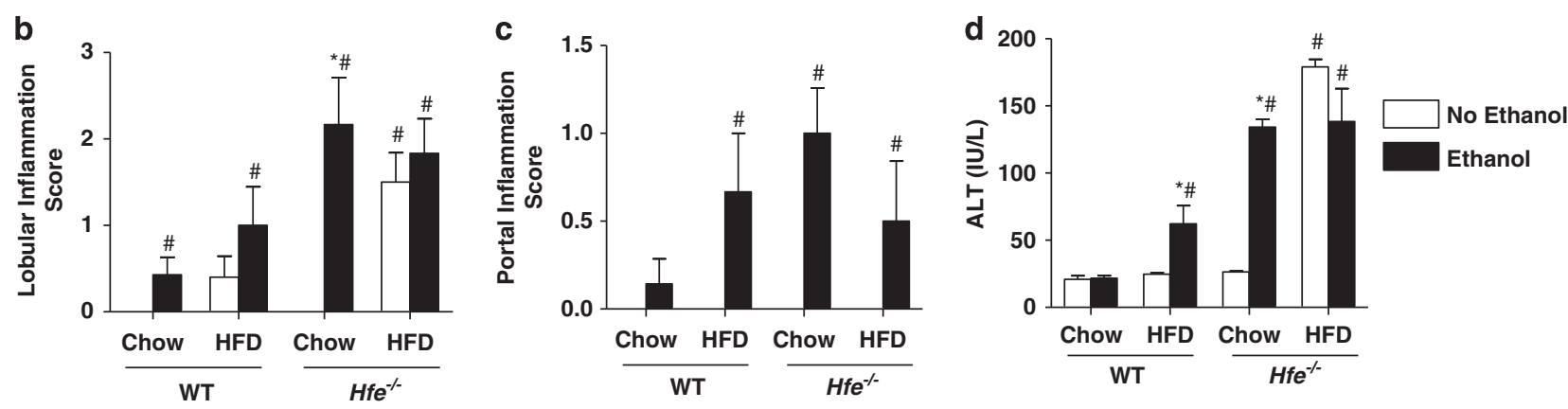

Figure 2 Assessment of liver injury. (a) Liver injury and steatosis as assessed by H\&E staining ( $\times 400$ magnification). (b) Histological semiquantitative assessment of lobular and (c) portal inflammatory scores and (d) serum ALT concentrations. ${ }^{\#} P<0.05$ compared with WT mice on chow only. ${ }^{*} P<0.05$ compared with nonethanol-treated animals within the same genotype and dietary group.

activates Ppar- $\alpha$, but this upregulation was attenuated in the $\mathrm{Hfe}^{-/-}$mice, despite almost twofold increases in circulating serum total adiponectin concentrations (Figure $6 f$ and Table 1).

Consistent with the evidence for increased lipogenic transcriptional activity and partial inhibition of fatty acid oxidation, p-AMPK $\alpha$ protein levels were markedly increased with HFD, with or without ethanol in the WT groups, but were significantly reduced with iron and ethanol (Figure 6g). The $H f^{-1-}$ mice also had a markedly attenuated increase in p-AMPK $\alpha$ following a HFD with or without ethanol. AMPK $\alpha$ is a key enzymatic regulator in fatty acid transport, and subsequent oxidation and a significantly reduced level suggests impaired fatty acid oxidation. This is reflected in the protein levels of the peroxisomal fatty acid $\beta$-oxidation enzyme acyl-coA oxidase 1 (ACOX1, Figure 6g), which showed reduction following co-administration in $\mathrm{Hfe}^{-/-}$ mice as compared with WT counterparts. Interestingly, cofeeding led to increased levels of Akt and phosphoinositide- dependent kinase-1 (PDK-1) in the WT mice (Figure 6h). Akt is a known negative regulator of AMPK and a stimulator of hepatic lipogenesis. However, the effect of iron on animals treated with HFD and ethanol showed a reduction in the protein levels of both kinases. PDK-1 is a master kinase that acts downstream of Akt and is a known activator of AMPK. Its function is crucial for the role of AMPK in fatty acid oxidation and lipid handling. The alterations in Akt and PDK-1 in the $\mathrm{Hfe}^{-1-}$ animals suggest that pathways upstream of AMPK are involved in iron-mediated modulation of ethanol- and HFD-induced hepatic lipid metabolism.

\section{Iron Partially Impairs Ethanol Metabolism and Exacerbates Oxidative Stress in Ethanol- and HFD- Induced Hepatotoxicity}

Given that oxidative stress is a common mechanism for ALD, NAFLD, and iron-induced hepatotoxicity, we investigated the effects of iron on markers of oxidative stress in the liver following ethanol and HFD consumption. The ratio of the 


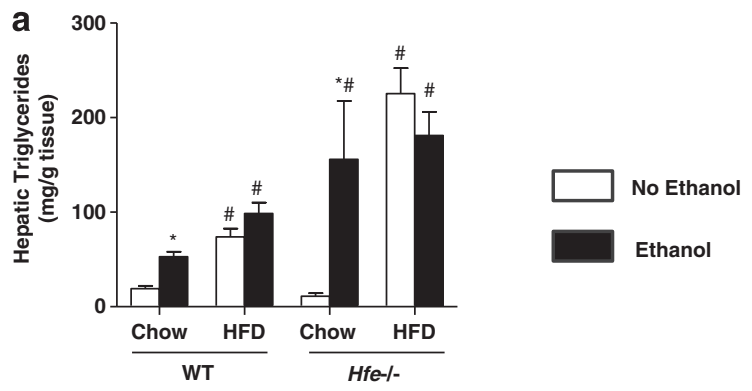

b
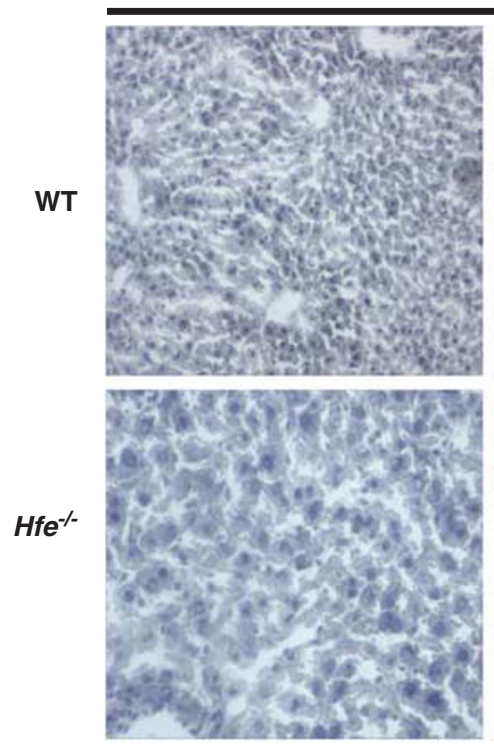

-
Chow
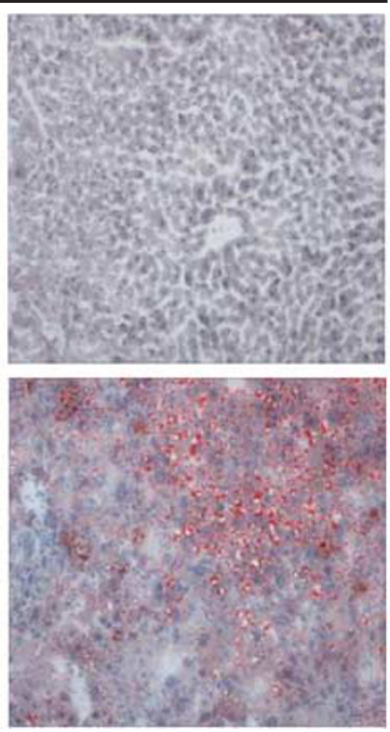

$+$
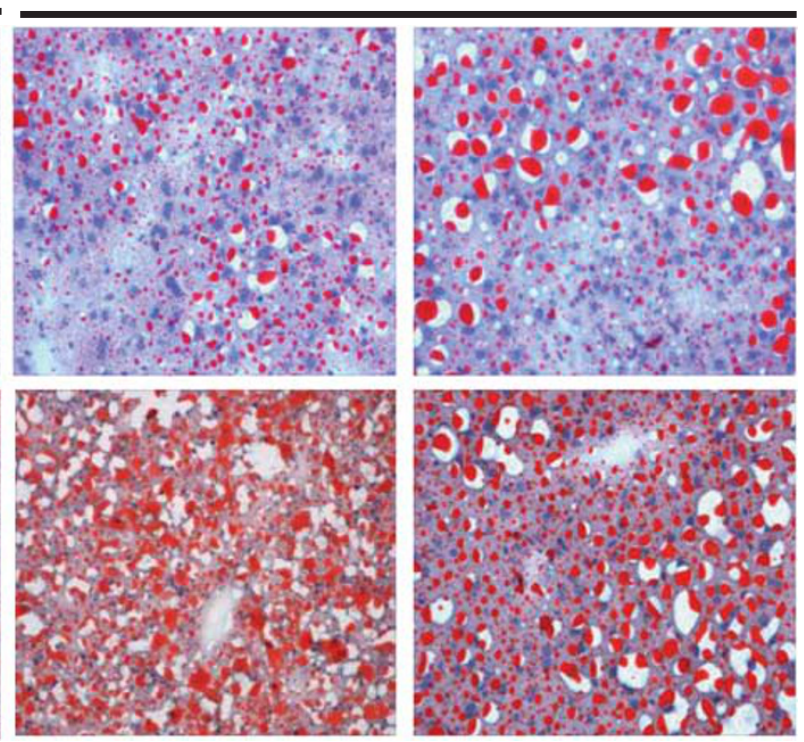

$+$

ETHANOL

Figure 3 Assessment of hepatic lipid content. (a) Hepatic triglyceride concentrations and (b) Oil Red O staining for hepatic triglycerides $(\times 200$ magnification). ${ }^{\#} P<0.05$ compared with WT mice on chow only. ${ }^{*} P<0.05$ compared with nonethanol-treated animals within the same genotype and dietary group.

antioxidant-reduced glutathione (GSH) to its oxidized form GSSG (GSH/GSSG) was not significantly affected by HFD or ethanol, either alone or in combination. Similarly, an increased hepatic iron in isolation did not alter this ratio significantly. However, the ratio was lowest when HFD was combined with iron, with or without ethanol, indicating a significant depletion in the antioxidant GSH and increased oxidative stress (Figure 7a). HFD or ethanol either alone or in combination led to reductions in mitochondrial antioxidant enzyme MnSOD (Figure 7b) and respiratory complex 1 activities (Figure 7c). However, these reductions were most pronounced (fivefold) following the combination of iron, $\mathrm{HFD}$, and ethanol in the $\mathrm{Hfe}^{-I_{-}}$animals.

As reported earlier, we noted the $\mathrm{Hfe}^{-/-}$groups fed ethanol had significantly higher serum ethanol levels compared with their WT controls fed the same diet. We hypothesized that increased iron may play a role in modulating ethanol metabolism in the liver. We measured the protein levels of two key alcohol metabolizing enzymes, alcohol dehydrogenase 1 (ADH1) and aldehyde dehydrogenase 1A1 (ALDH1A1), the key cytosolic metabolizer of acetaldehyde (by-product of alcohol metabolism by ADH1). Paradoxically, the $\mathrm{ADH} 1$ protein was significantly reduced by ethanol in the WT animals fed chow (Figure 7d). However, this effect was reversed by the effect of HFD. The increased iron did not appear to alter the protein levels of these enzymes significantly. Surprisingly, we did not observe any effect of iron, ethanol, or HFD on the protein levels of ALDH1A1. However, when we measured the transcript levels of $A d h 1$ and the mitochondrial acetaldehyde metabolizing enzyme, Aldh2, the presence of iron overload appears to impair the gene expression of these two hepatic enzymes (Figures $7 \mathrm{e}$ and $\mathrm{f}$ ). Interestingly, a HFD also appears to have a negative effect on the mitochondrial Aldh2 mRNA expression, whereas increased iron caused further decreases in Aldh2 mRNA levels in the presence of ethanol (Figure 7f). 
a

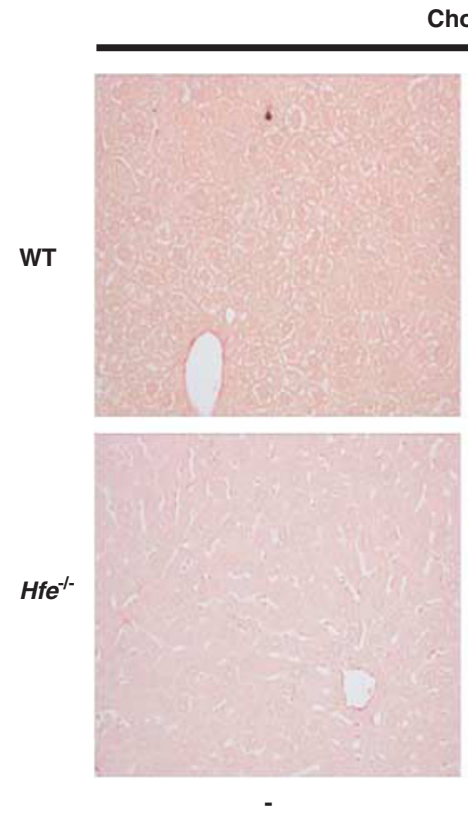

Chow
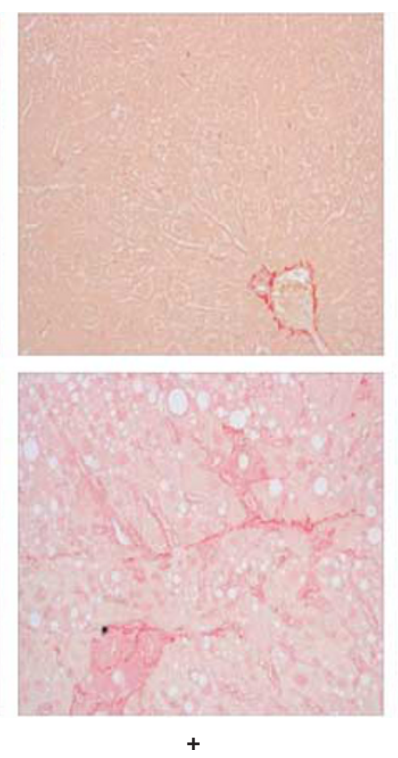

HFD
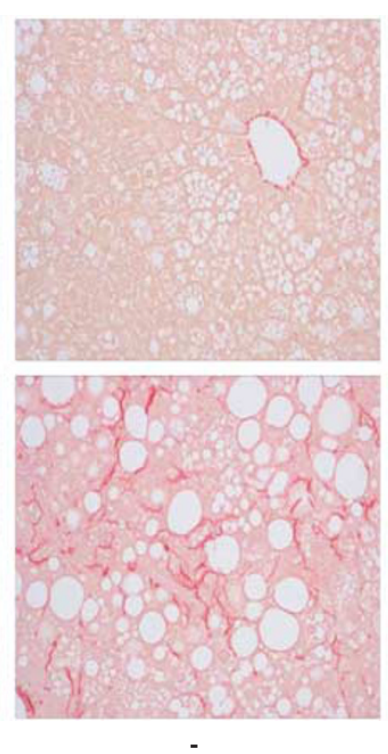
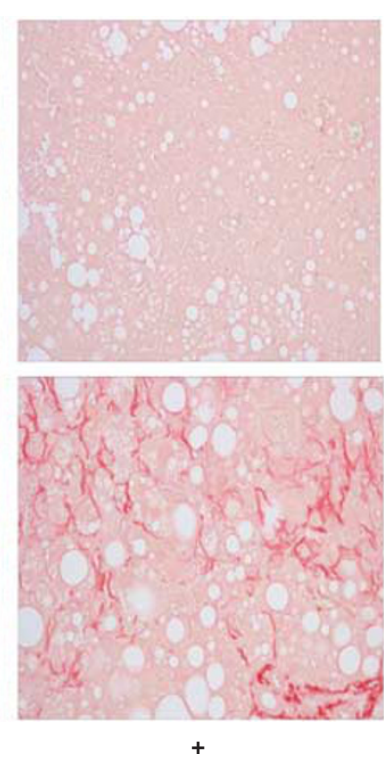

ETHANOL

b
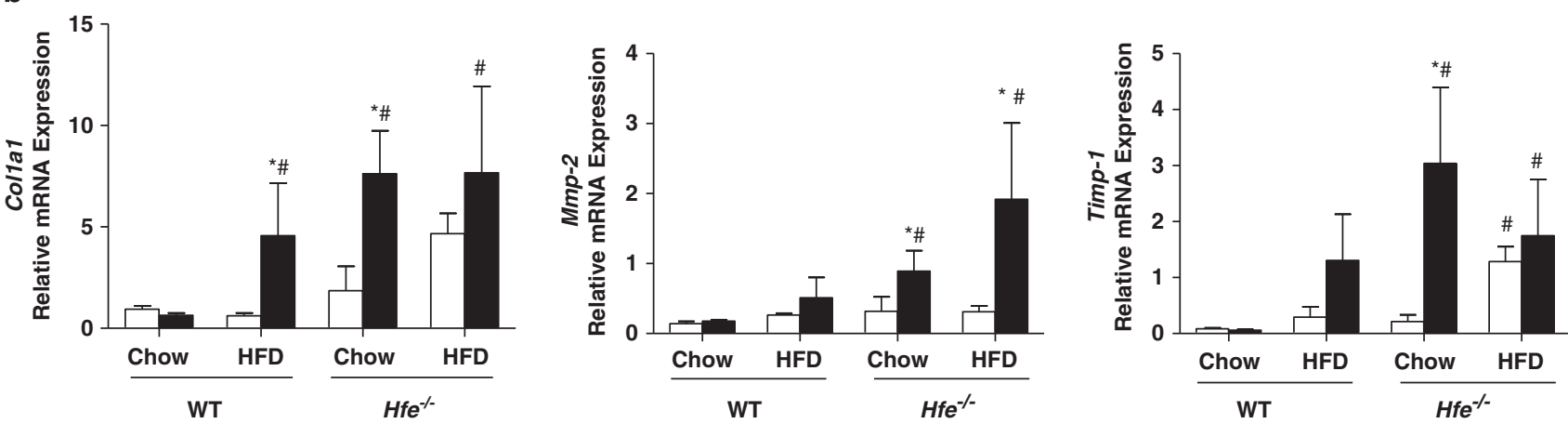

Figure 4 Advanced hepatic fibrosis with iron, ethanol, and high-fat diet. (a) Sirius Red staining for collagen deposition ( $\times 400$ magnification) revealed portal-portal tract linkage fibrosis in Hfe ${ }^{-1-}$ mice on ethanol and HFD. (b) RT-PCR analysis for genes involved in fibrogenesis (Colla1, Mmp-2, and Timp-1). ${ }^{\#} P<0.05$ compared with WT mice on chow only. ${ }^{*} P<0.05$ compared with nonethanol-treated animals within the same genotype and dietary group.

Another critical enzyme involved with hepatic ethanol metabolism is the microsomal pro-oxidant cytochrome $P 450$ CYP2E1. Ethanol feeding expectedly led to an increase in the protein levels of CYP2E1 in the WT mice. This was increased further following HFD and ethanol (Figure 7d). However, the corresponding increases were markedly attenuated with hepatic iron overload (Figure $7 \mathrm{~d}$ ), suggesting that iron may in fact partially impair ethanol metabolism by the CYP2E1 pathway. The lipid peroxidation by-product 4-hydroxynonenal (4-HNE) was significantly increased in the WT mice on ethanol and HFD as well as $\mathrm{Hfe}^{-1-}$ animals on chow and ethanol (Figure 7d). However, the addition of HFD in the $\mathrm{HFe}^{-/-}$groups had no further effect in increasing the levels of these protein adducts. These surprising results were also reflected in the TBARS assay, where levels of MDA were highest in the $\mathrm{Hfe}^{-1-}$ group on chow diet treated with ethanol, but not in the HFD-fed $\mathrm{Hfe}^{-/-}$groups (Figure 7g), suggesting that other mechanisms independent of lipid peroxidation and oxidative were involved in iron-mediated combined hepatotoxicity here.

\section{Iron Overload Augments Ethanol- and HFD-Induced Activation of Proinflammatory Mediators and Toll-Like Receptor Signaling}

We next sought to determine the potential mechanisms underlying the iron-induced increase in liver injury caused by ethanol and/or HFD by measuring the gene expression of key proinflammatory mediators and cytokines. Alcohol and HFD can increase bacterial translocation in the gut, with subsequent portal endotoxemia and stimulation of the pattern recognition TLR pathways. ${ }^{17,26}$ Induction of the TLRs can lead to hepatic macrophage activation and production of 
a
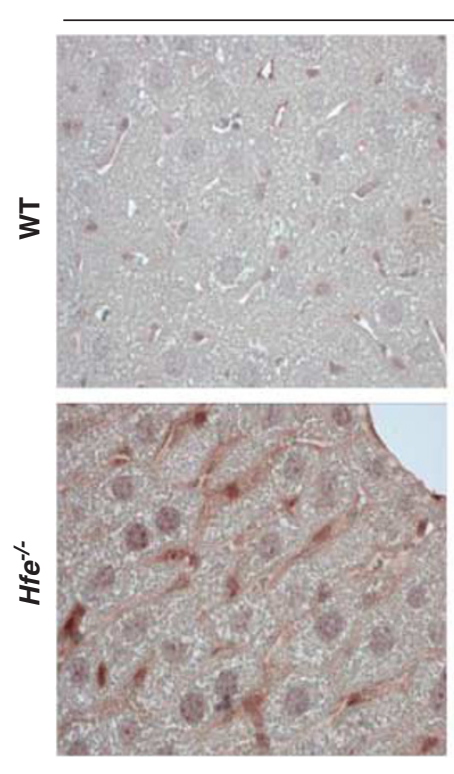

Chow
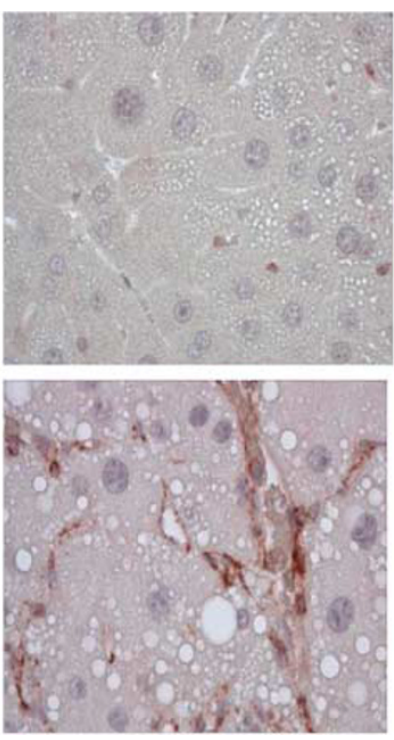

$+$
HFD
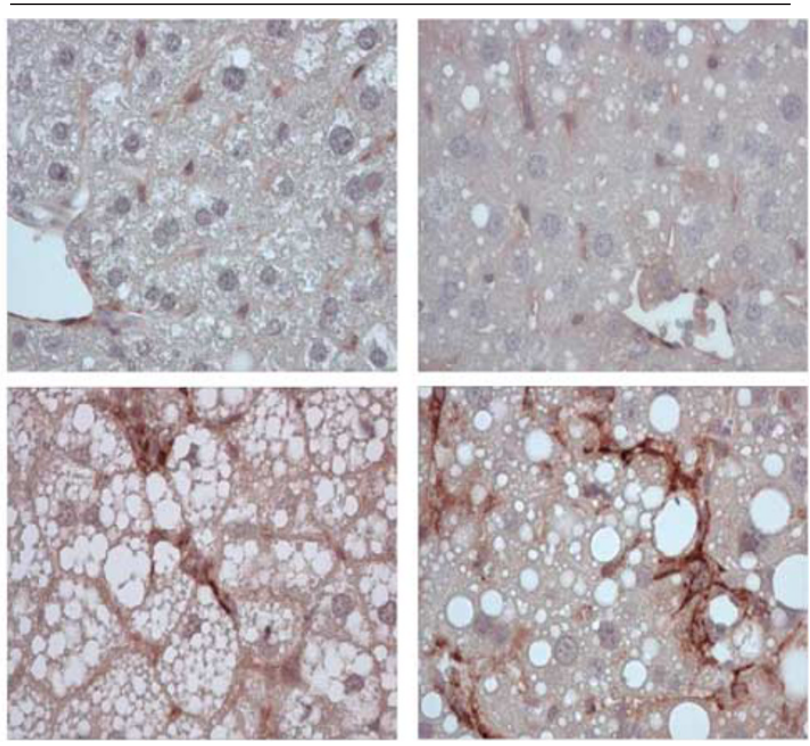

-

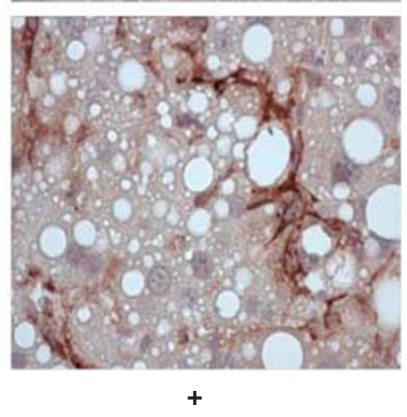

ETHANOL
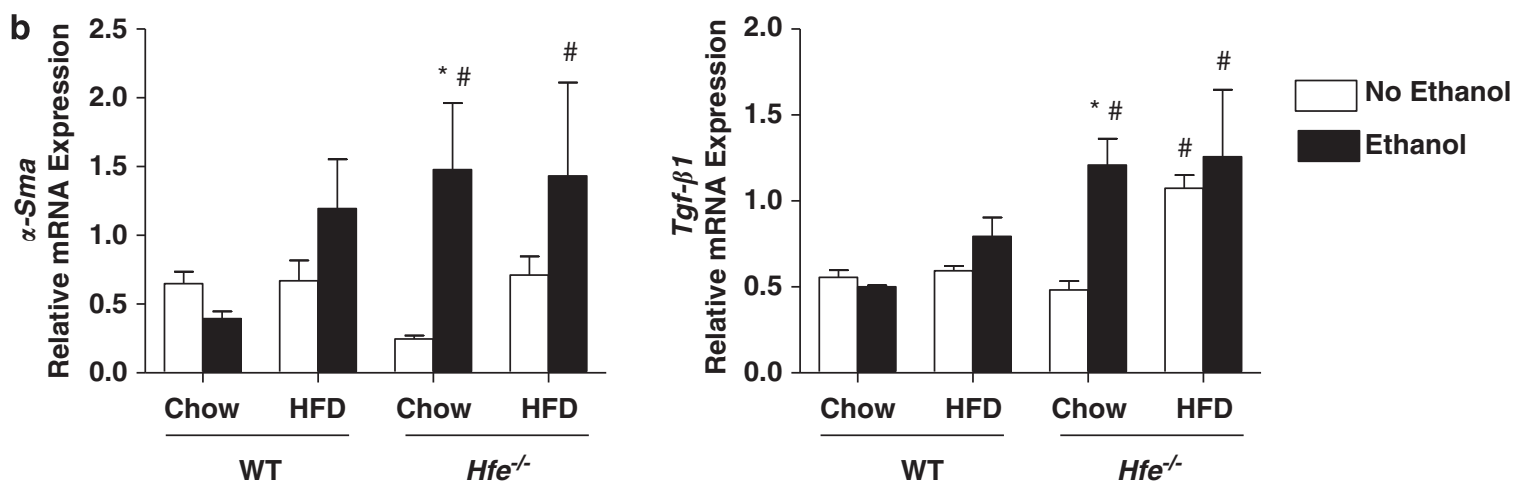

Figure 5 Assessment of hepatic stellate cell activation. (a) Immunohistochemistry for $\alpha$-SMA ( $\times 400$ magnification) and (b) RT-PCR analysis for genes indicative of hepatic stellate cell activation ( $\alpha$-Sma and Tgf- $\beta 1) .{ }^{\#} P<0.05$ compared with WT mice on chow only. ${ }^{*} P<0.05$ compared with nonethanoltreated animals within the same genotype and dietary group.

Kupffer cell-derived tumor necrosis factor- $\alpha(\mathrm{TNF}-\alpha)$ and monocyte chemoattractant protein-1 (MCP-1). ${ }^{27}$ HFD or ethanol in isolation had no effect on the gene expression of Tnf- $\alpha, M c p-1$ (downstream target of Tnf- $\alpha$ ), or hepatocyte growth factor $(H g f)$, although there was a moderate increase in these genes with combination feeding (Figures $8 \mathrm{a}-\mathrm{c}$ ). However, the combination of increased iron and ethanol (regardless of diet) led to a 10-fold increase in Tnf- $\alpha$ mRNA expression (Figure 8a) over WT chow controls. This is also associated with a significant mRNA upregulation of Mcp-1 (Figure $8 \mathrm{~b}$ ) and of the prosurvival and antiapoptotic $\mathrm{Hgf}$ (Figure $8 \mathrm{c}$ ), as well as the protein levels of the mitogenactivated kinase extracellular signal-regulated kinase, ERK1/2 (p-ERK1/2) (Figure 8d). ERK1/2 is known to promote cell growth and repair through a TNF- $\alpha$-dependent mechanism. The induction of Tnf- $\alpha$ and its associated downstream targets are associated with an upregulation of multiple TLR genes, known to be increased by Tnf- $\alpha$, including Tlr2, Tlr3, Tlr4, $\operatorname{Tlr} 5$, and $\operatorname{Tl} 7$ (Figures $8 \mathrm{e}-\mathrm{h}$ ).

\section{Endoplasmic Reticulum Stress and Apoptotic Signaling in Combined Iron-, Ethanol-, and Obesity-Induced Fibrosing Steatohepatitis}

To understand the contribution of iron toxicity to ethanoland diet-induced liver injury, we investigated the possibility that ER stress may contribute to its pathogenesis. ER stress has been linked to the development of ALD and NAFLD and may be a contributor toward hepatic injury and inflammation. The protein levels of two key markers of ER stress, CCAAT/ enhancer binding protein-homologous protein (CHOP) (Figure 9a) and phosphorylated protein kinase doublestranded RNA-dependent-like ER kinase (p-PERK) 

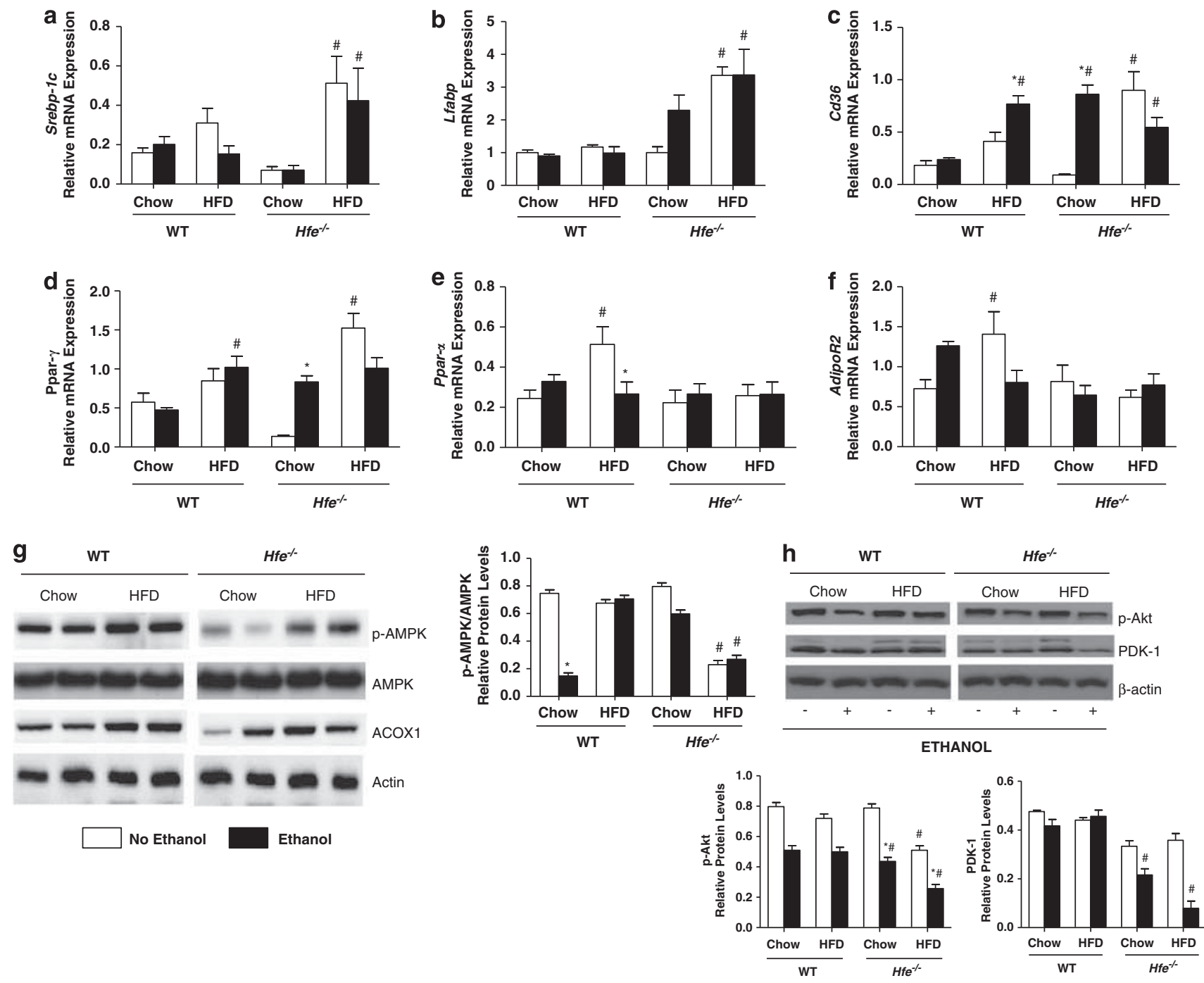

Figure 6 Iron overload impairs hepatic lipid handling following ethanol and high-fat diet. RT-PCR analysis for genes involved in hepatic (a) lipogenesis $($ Srebp-1c), (b) fatty acid entry (Lfabp), (c) trafficking (Cd36), (d) storage (Ppar- $\gamma$ ) and fatty acid $\beta$-oxidation, (e) Ppar- $\alpha$, and (f) AdipoR2. Representative western blots and quantitative analysis for proteins involved in lipid metabolism including (g) p-AMPK and ACOX1 (h) and p-Akt and PDK1. The $\beta$-actin levels indicate protein loading. ${ }^{\#} P<0.05$ compared with WT mice on chow only. ${ }^{*} P<0.05$ compared with nonethanol-treated animals within the same genotype and dietary group.

(Figure 9b), were highest in HFD-fed $\mathrm{Hfe}^{-/-}$mice, with and without ethanol. Interestingly, although ethanol and HFD increase the glucose-regulated protein 78 (GRP78) levels in the WT groups, the presence of increased hepatic iron had the opposite effect in the $\mathrm{Hfe}^{-/-}$mice, likely reflecting the dissociation of this molecular chaperone protein from the UPR transducers and its subsequent sequestration (Figure 9c). However, despite the evidence for increased PERK activation, the phosphorylation of the protein eukaryotic initiation factor $2-\alpha$ (p-EIF2 $\alpha)$, which is involved in the attenuation of protein synthesis following increased protein load, was not increased. In the WT animals, a combination of HFD and ethanol led to increased phosphorylated (activated) inositol-requiring enzyme-1 $\alpha$ (p-IRE1 $\alpha$ ) (Figure 9d), a key regulator of the unfolded protein response initiation, and the subsequent splicing and activation of X-box binding protein-1 (sXBP1), one of its key transduction targets (Figure 9e). This process was intensified in the $\mathrm{Hfe}^{-/-}$mice, with marked increases in activated IRE1 $\alpha$ and spliced XBP1. The protein levels of protein disulfide isomerase (PDI) was highest in ironoverloaded mice fed ethanol and chow, but a HFD had no additional effects in increasing the expression of this protein misfolding chaperone (Figure 9b). The mRNA expression for the ER stress molecular chaperone Grp94 was significantly suppressed with combined iron and ethanol, with or without HFD (Figure 9f).

As one of the downstream effects of pathologic ER stress and prolonged UPR is apoptosis, we evaluated some key markers of apoptotic signaling in the hepatic lysates. HFD in combination with ethanol increased the mRNA expression of 


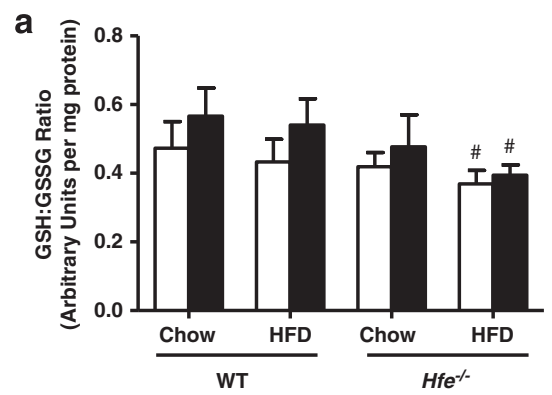

d

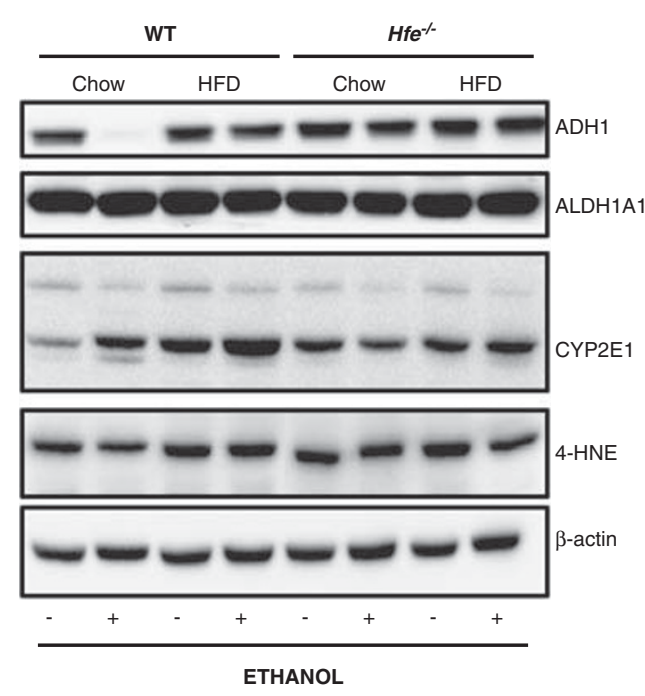

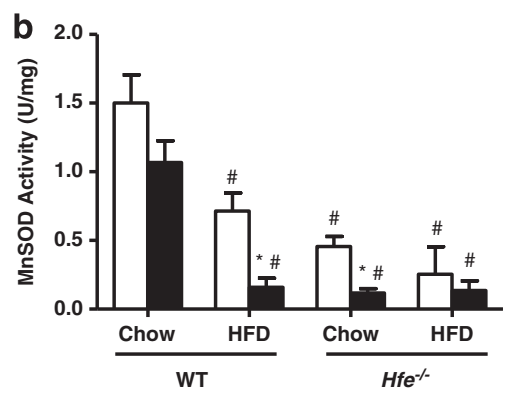

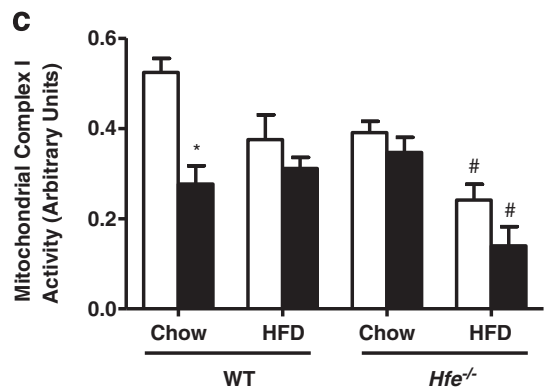

e
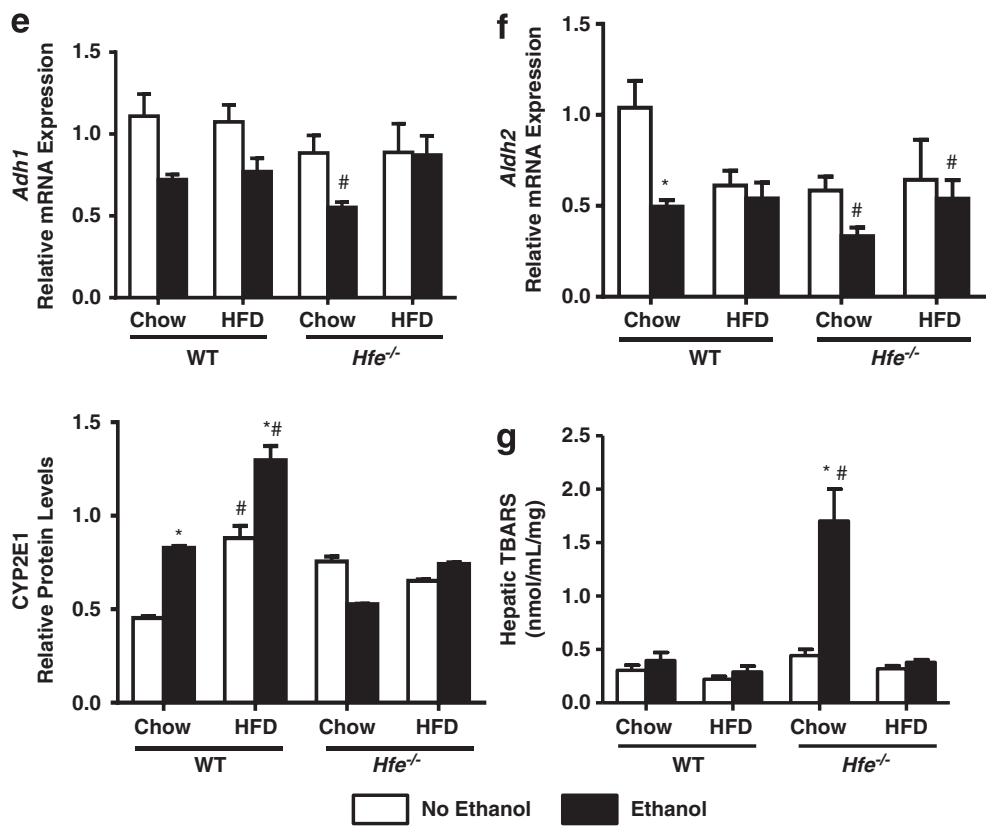

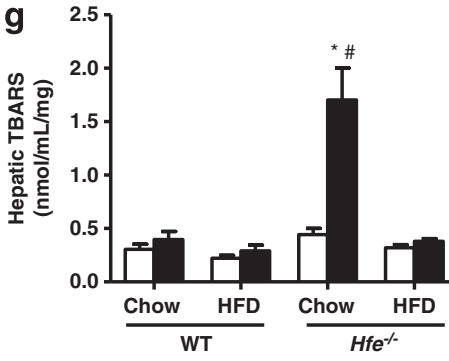

Ethanol

Figure 7 Markers of oxidative stress and mitochondrial dysfunction. (a) Ratio of reduced (GSH)-to-oxidized (GSSG) forms of glutathione (b) mitochondrial MnSOD and (c) mitochondrial respiratory complex 1 activities. (d) Representative western blots for ADH1, ALDH1A1, CYP2E1, and 4-HNE. (e) RT-PCR analysis for alcohol and aldehyde metabolizing enzymes Adh1 and (f) Aldh2 mRNA. (g) Hepatic MDA levels as per TBARS measurements. ${ }^{\#} P<0.05$ compared with WT mice on chow only. ${ }^{*} P<0.05$ compared with nonethanol-treated animals within the same genotype and dietary group.

proapoptotic proteins Bax (Figure 10a) and Bcl-2 (Figure 10b), and this increase was magnified by iron excess. Consistent with these transcript-level increases, the numbers of apoptotic cells in the liver were most marked with the combination of HFD, ethanol, and iron, as seen on the TUNEL assay (Figure 10c). None of the WT mice treated with HFD or ethanol, either alone or in combination, or $\mathrm{Hfe}^{-/-}$ mice on chow-only diet displayed any evidence of hepatic apoptosis. Surprisingly, when we investigated proteins of the caspase pathways, the levels of caspases 3, 9, and 12 were not significantly altered (Supplementary Figure S6). Surprisingly too, the activation of $\mathrm{p}$-JNK was decreased with combined iron, ethanol, and HFD in the $H f e^{-/-}$groups as compared with WT animals, which showed a moderate increase in response to HFD (Figure 10d), suggesting that JNK may actually play a protective role in the synergistic liver injury caused by these three cofactors and may be inactivated by excess iron.

\section{Iron-Mediated Synergistic Ethanol and Obesity-Induced Hepatotoxicity Is Associated with Impaired Hepatic Autophagy}

Consistent with an impaired UPR and increased ER stress, we found evidence of impaired autophagy responses in the Hfe $\mathrm{e}^{-/-}$ but not WT mice. A by-product of impaired autophagy in cells is the accumulation of the ubiquitin-binding scaffold protein, the sequestosome 1 p62 (Figure 11a). P62 is degraded by activation of autophagy and serves to link ubiquitinated proteins to the autophagy machinery for degradation. Levels of this protein were highest in the mice with combined iron-, ethanol-, and HFD-induced hepatotoxicity and were associated with a marked reduction in one of the key proteins of the autophagic response in the $\mathrm{Hfe}^{-/-}$mice, such as beclin-1, a regulator of autophagosome formation (Figure 11b). Similarly, the expression of the microtubule protein 1 light chain 3 (LC3-I and LC3-II), markers of autophagosome numbers and autophagic flux, were also altered with the effects of ethanol, diet, and 


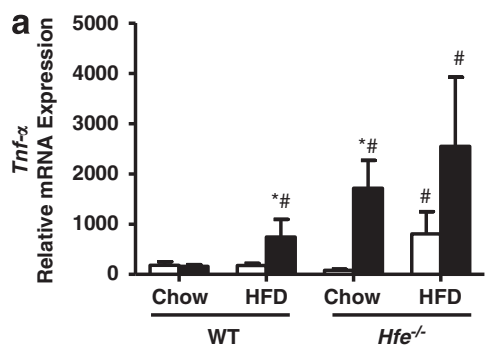

d
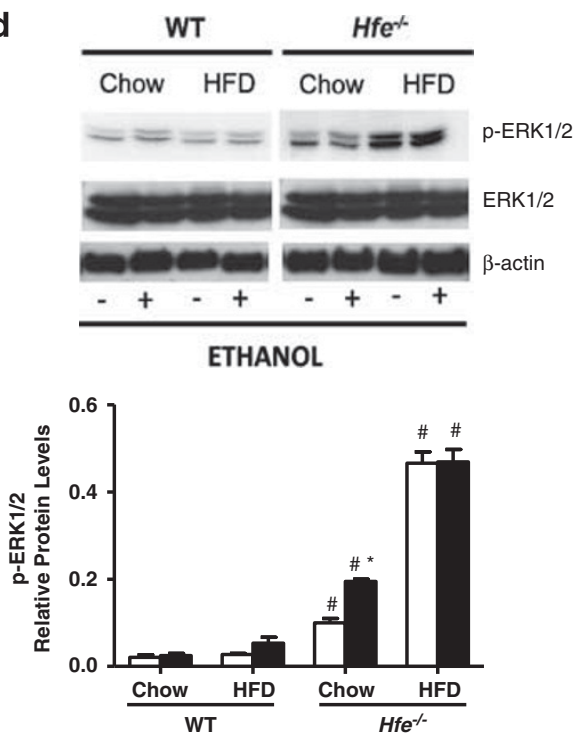

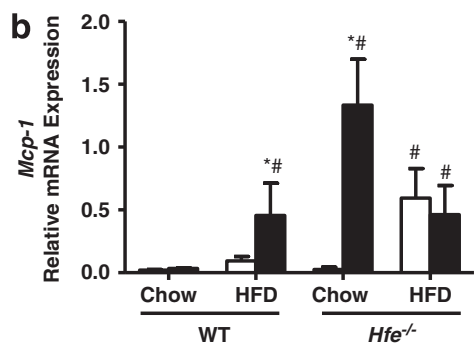

e

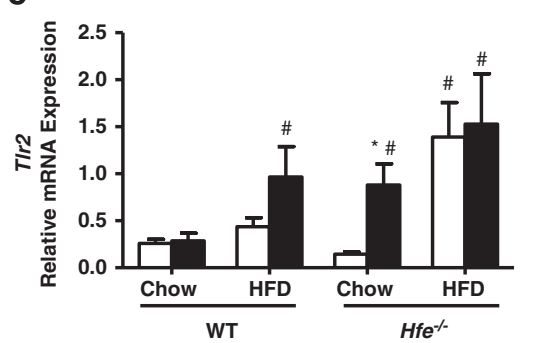

$\mathbf{g}$ 으

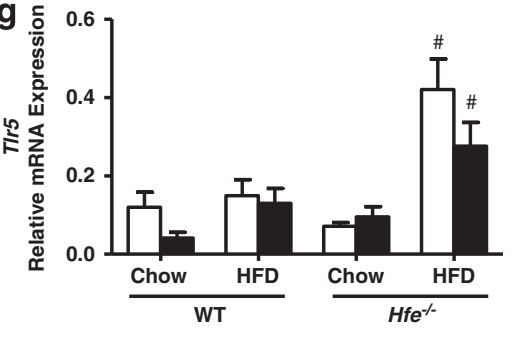

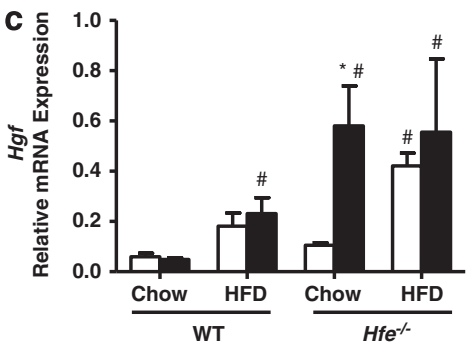

f
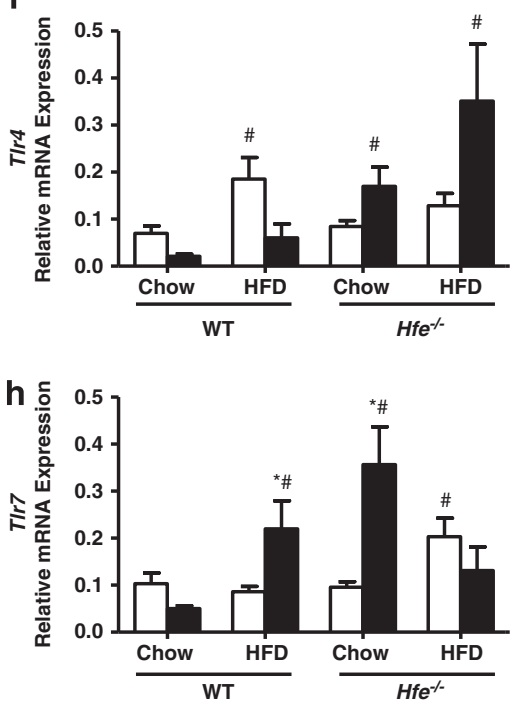

No Ethanol

Ethanol

Figure 8 Markers of proinflammatory and Toll-like receptor (TLR) signaling pathways. RT-PCR analysis for macrophage activation including (a) Tnf- $\alpha$, (b) Mcp-1, and (c) Hgf. (d) Representative western blot and quantitative analysis for prosurvival protein mitogen-activated protein kinase $\mathrm{p}$-ERK1/2 and RT-PCR analysis for TLR genes including (e) T/r2, (f) T/r4, (g) T/r5, and (h) T/r7. ${ }^{*} P<0.05$ compared with WT mice on chow only. ${ }^{*} P<0.05$ compared with nonethanol-treated animals within the same genotype and dietary group.

the presence of iron (Figure 11c). Ethanol consumption in the WT mice increased the amount of the cytosolic LC3-I, whereas the combination of ethanol and HFD led to an increase in its conjugation to LC3-II. However, iron overload caused a decrease in LC3-I with a subsequent decrease in the level of its conjugation to LC3-II, indicating a potential impairment in the activity of autophagic flux induced by iron. Consistent with the defective autophagic flux, the protein levels of autophagyrelated genes Atg 3, 5, and 7 were reduced with iron excess and co-feeding (Figures 11d and e). To understand the reason for the lack of Atg and LC3 recruitment in the autophagic process in iron-overloaded co-fed mice, we assessed the protein levels of eIF2 $\alpha$, an ER stress protein and known activator of the Atg5-12 complex, ${ }^{28}$ and found no increase in the activated form of this protein (p-eIF2 $\alpha$ ) in the $\mathrm{Hfe}^{-/-}$animals (Figure 9c).

\section{DISCUSSION}

The purpose of this study was to establish an easy-to-use, readily reproducible, small-animal experimental model of advanced liver injury using the combined effects of com- monly seen co-toxins in human disease; namely iron, alcohol, and HFD. This model allowed us to study the mechanisms pertaining to interactive effects of each hepatotoxic cofactor. A second aim of this study was to further investigate the mechanisms by which iron interacts with ethanol and steatosis to further exacerbate liver disease. The effects of iron on oxidative stress and lipid peroxidation are well documented, but it remains unclear whether other mechanisms are involved, such as ER stress and related pathways. The iron excess seen in our animal model of hemochromatosis was mild to moderate that typically resembles the magnitude of iron excess seen in early HFE hemochromatosis- or nonhemochromatosis-related iron overload such as that seen in ALD and NAFLD/NASH. ${ }^{29}$ This model also recapitulates features essential for the 'synergistic liver injury effect' paradigm of common hepatic co-toxins in CLD. ${ }^{15,30}$

In this study, we found that iron excess affects multiple aspects of the cellular stress signaling pathways previously described in ALD and NAFLD/NASH. We showed that iron overload may lead to an impairment in hepatic cellular stress responses that regulate the development of steatosis, 
a

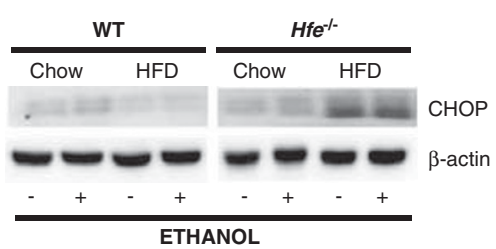

b

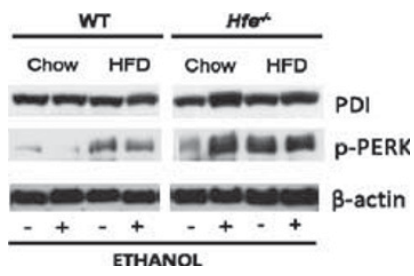

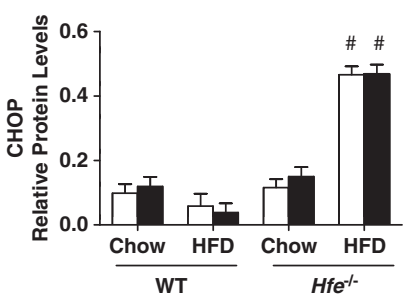

C

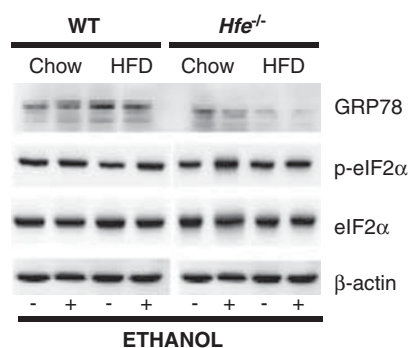

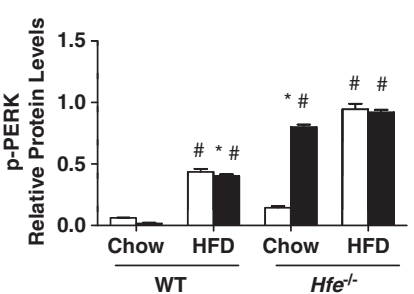
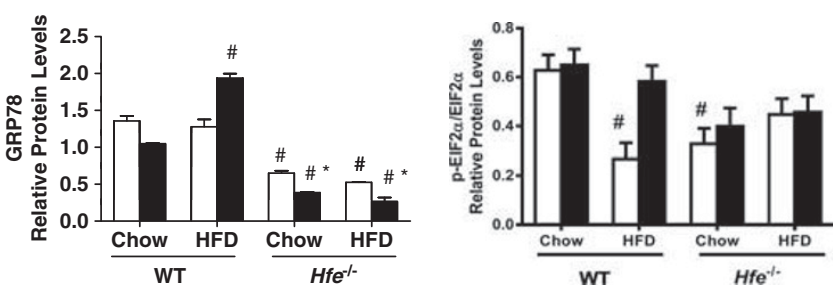

d

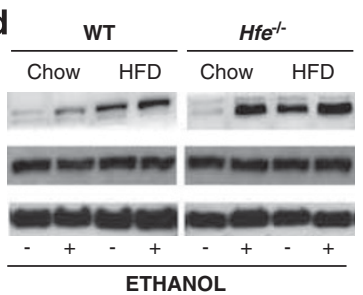

e
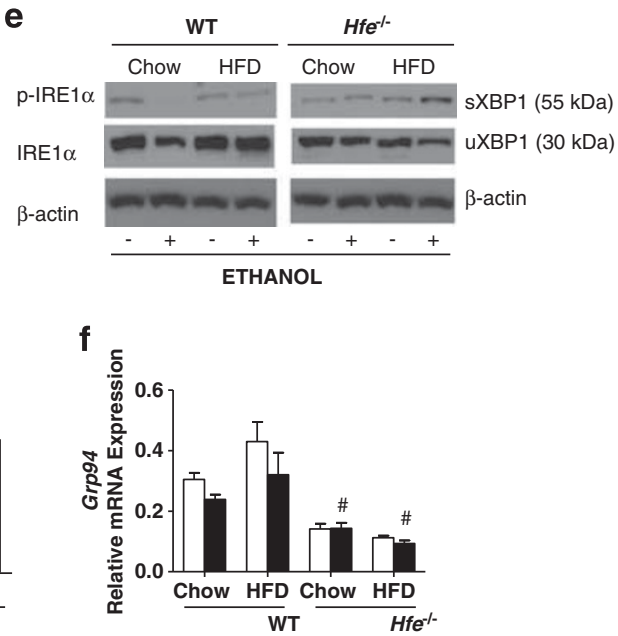

$\square$ No Ethanol $\square$ Ethanol

Figure 9 Iron overload enhances ER stress and modulates the unfolded protein response in alcohol- and obesity-induced liver injury. Representative western blots and quantitative analysis for proteins involved in the UPR and ER stress including (a) CHOP, (b) PDI and p-PERK, (c) GRP78, p-EIF2 $\alpha$, and EIF2 $\alpha$, (d) p-IRE1 $\alpha$ and IRE1 $\alpha$, and (e) spliced (sXBP1) and unspliced (uXBP1) forms of XBP1. (f) RT-PCR analysis for molecular chaperone Grp94. The $\beta$-actin levels indicate protein loading. ${ }^{\#} P<0.05$ compared with WT mice on chow only. ${ }^{*} P<0.05$ compared with nonethanol-treated animals within the same genotype and dietary group.

oxidative stress, inflammation, and apoptosis. ${ }^{6}$ Two of such pathways of interest are the UPR and autophagy.

Prolonged ER stress is linked to lipogenesis, oxidative stress, inflammation, and activation of apoptotic pathways, ${ }^{31}$ and has been implicated in the pathogenesis of ALD and NAFLD. ${ }^{14,32}$ However, it remains unclear whether iron has any role in promoting ER stress-associated liver injury in these disorders. In the absence of ethanol and/or HFD, iron excess in itself had no real effect on the UPR and ER stress proteins. Similarly, there were no significant effects of alcohol and HFD on this pathway in the absence of increased hepatic iron. However, in the presence of excess iron, the HFD, with or without alcohol, caused marked increases in the protein expression of ER stress markers such as $\mathrm{CHOP}$ and activated PERK, as well as a significant activation of the prosurvival protein ERK1/2 in response to increased liver injury and 

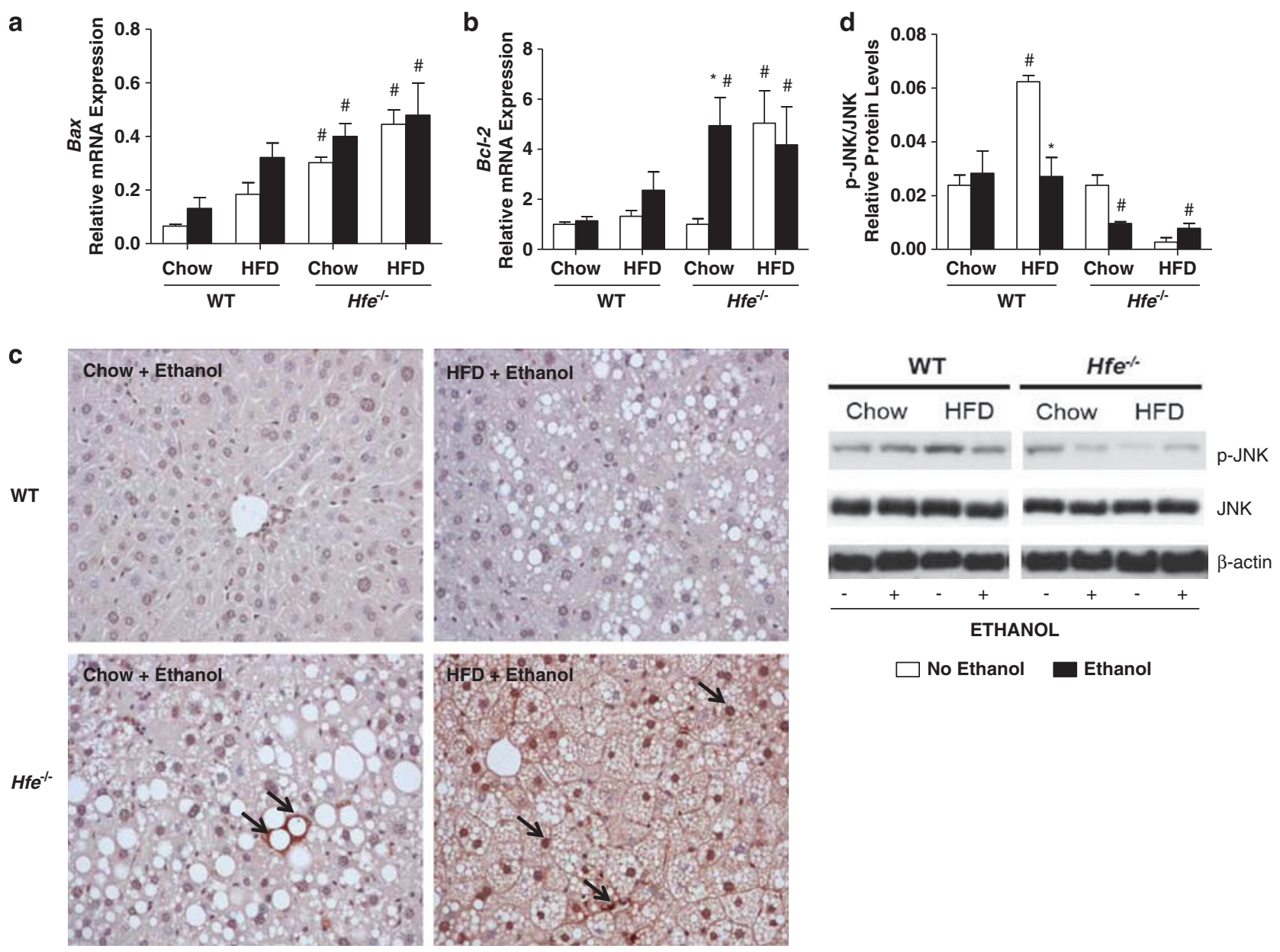

Figure 10 Markers of hepatocellular apoptosis. RT-PCR analysis for proapoptotic genes (a) Bax and (b) Bcl-2. (c) Representative sections of TUNEL assay comparing WT and $\mathrm{Hfe}^{-/-}$animals that were either co-fed chow and ethanol or HFD and ethanol and (d) representative western blots and quantitative analysis for $\mathrm{p}$-JNK and JNK. The $\beta$-actin levels indicate protein loading control. ${ }^{\#} P<0.05$ compared with WT mice on chow only. ${ }^{*} P<0.05$ compared with nonethanol-treated animals within the same genotype and dietary group. Arrows indicate apoptotic nuclei.

hepatocellular death. These changes are consistent with pathologic ER stress in the liver. Although a combination of HFD and ethanol feeding led to an increase in the activation of IRE $1 \alpha$ in the WT mice, this effect was magnified with iron excess, consistent with a subsequent increase in the levels of active spliced XBP1. The level of GRP78 was depleted, reflecting the importance of its binding activities to the UPR transducers, and its subsequent dissociation and sequestration with the activation of UPR. However, despite the presence of activation of certain proteins of the UPR, not all pathways were affected. Specifically, EIF $2 \alpha$ activation, which is downstream of PERK, was not increased, suggesting that certain elements of the UPR could be impaired by ironinduced ER toxicity. Given that the ER derives its energy from the mitochondria, it is plausible that iron-induced mitochondrial impairment may enhance ER dysfunction, suggesting an integral role for mitochondria-ER interaction in basic cellular homeostatic response to stress in health and disease.
Excessive production of ROS is implicated in the pathogenesis of ALD, NAFLD, and iron hepatotoxicity. ROS and lipid peroxidation products cause mitochondrial DNA damage, impaired mitochondrial function, ATP depletion, and necrosis. ROS signals to induce TNF- $\alpha$ and activate JNK1, both of which are implicated in hepatocellular apoptosis and development of steatohepatitis. ${ }^{33}$ To our surprise, the activity of JNK was suppressed in the livers with combined liver injury. Current evidence suggests that JNK activation may promote cellular injury and apoptosis, but others have found that its induction may be a mechanism of cellular adaptive response to chronic stresses. ${ }^{34}$ Given the role of IRE1 $\alpha$ in preventing ER stress-induced hepatic steatosis, the suppression of JNK may reflect its role in nonapoptotic stress signaling responses in the development of hepatic injury secondary to iron, ethanol, and obesity. ${ }^{35}$

To strengthen the argument for the role of iron in ER stress, we also examined pathways that are related to the UPR. A dysfunctional UPR may be a precursor for impaired autophagy, 
a

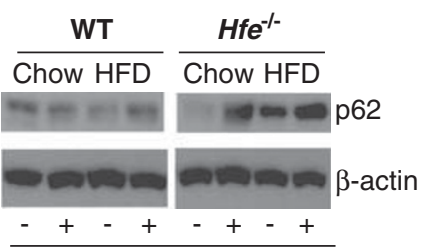

ETHANOL b

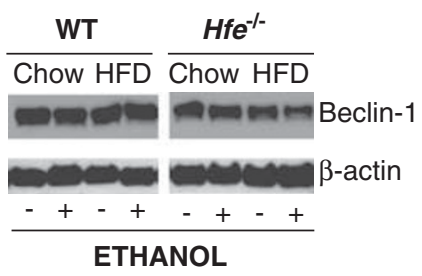

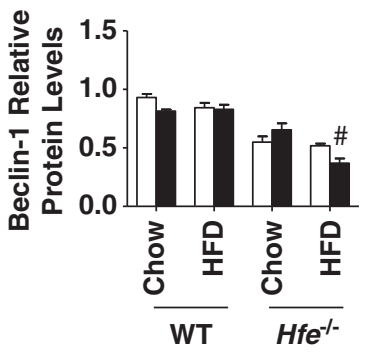

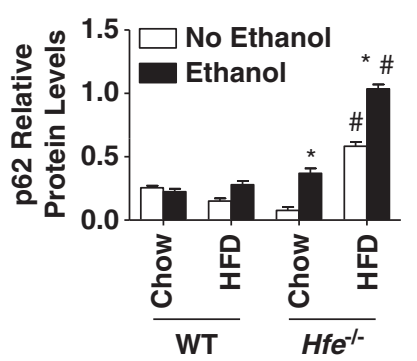

d

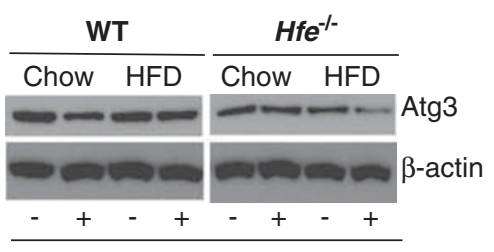

ETHANOL

C
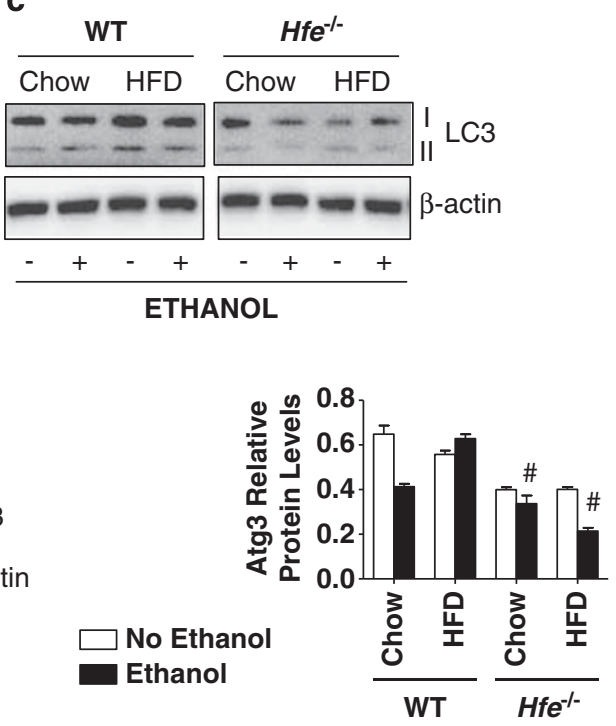

e
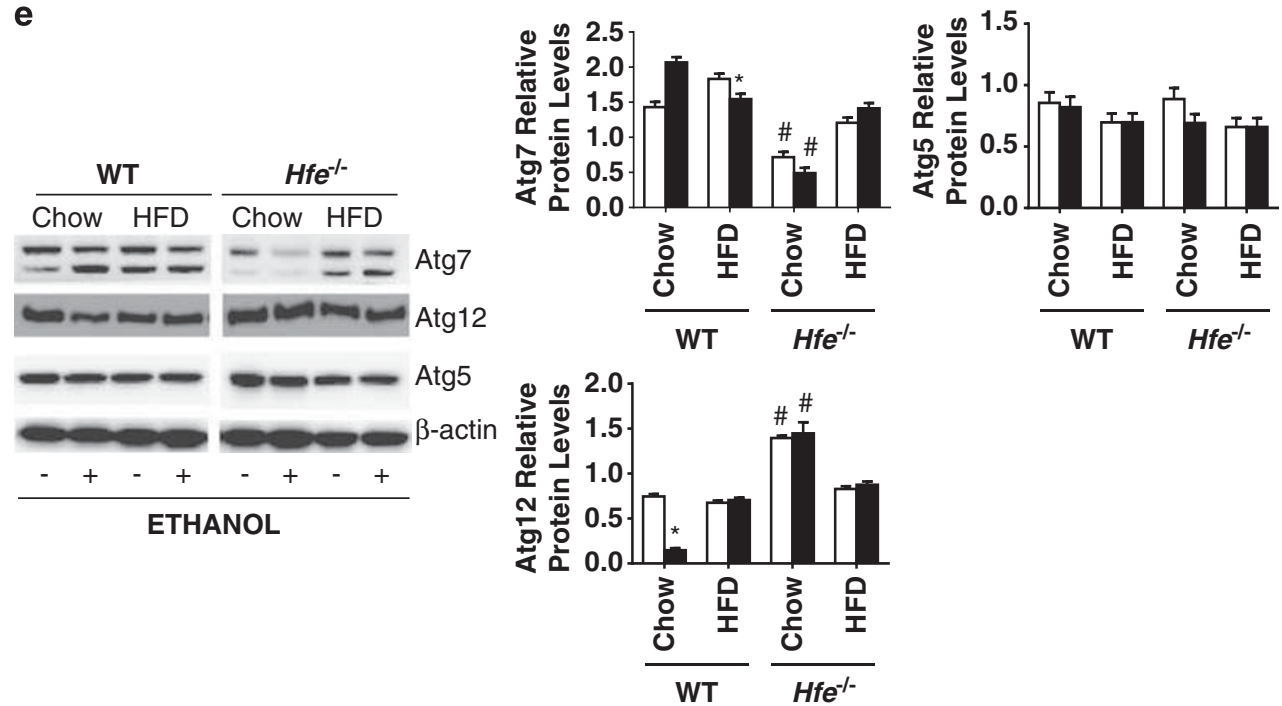

Figure 11 Assessment of autophagic function. Representative western blots and quantitative analysis for autophagy proteins (a) p62, (b) Beclin-1, (c) LC3-I and LC3-II, (d) Atg3, and (e) Atg 5, 7, and 12. The $\beta$-actin levels indicate protein loading control. " $P<0.05$ compared with WT mice on chow only. ${ }^{*} P<0.05$ compared with nonethanol-treated animals within the same genotype and dietary group.

disrupted containment of toxic cytosolic waste products, and subsequent activation of stress and proinflammatory signaling pathways. Autophagy is an intracellular lysosomal pathway that degrades and recycles intracellular organelles and proteins, and participates in the removal of damaged cell components. ${ }^{36}$ An adequate autophagic function is crucial in preventing liver injury. ${ }^{37}$ This ability of autophagy to regulate lipid metabolism and cellular stress responses, particularly death from oxidant stress and mitochondrial damage, suggests its deficiency might aggravate hepatic steatosis in 


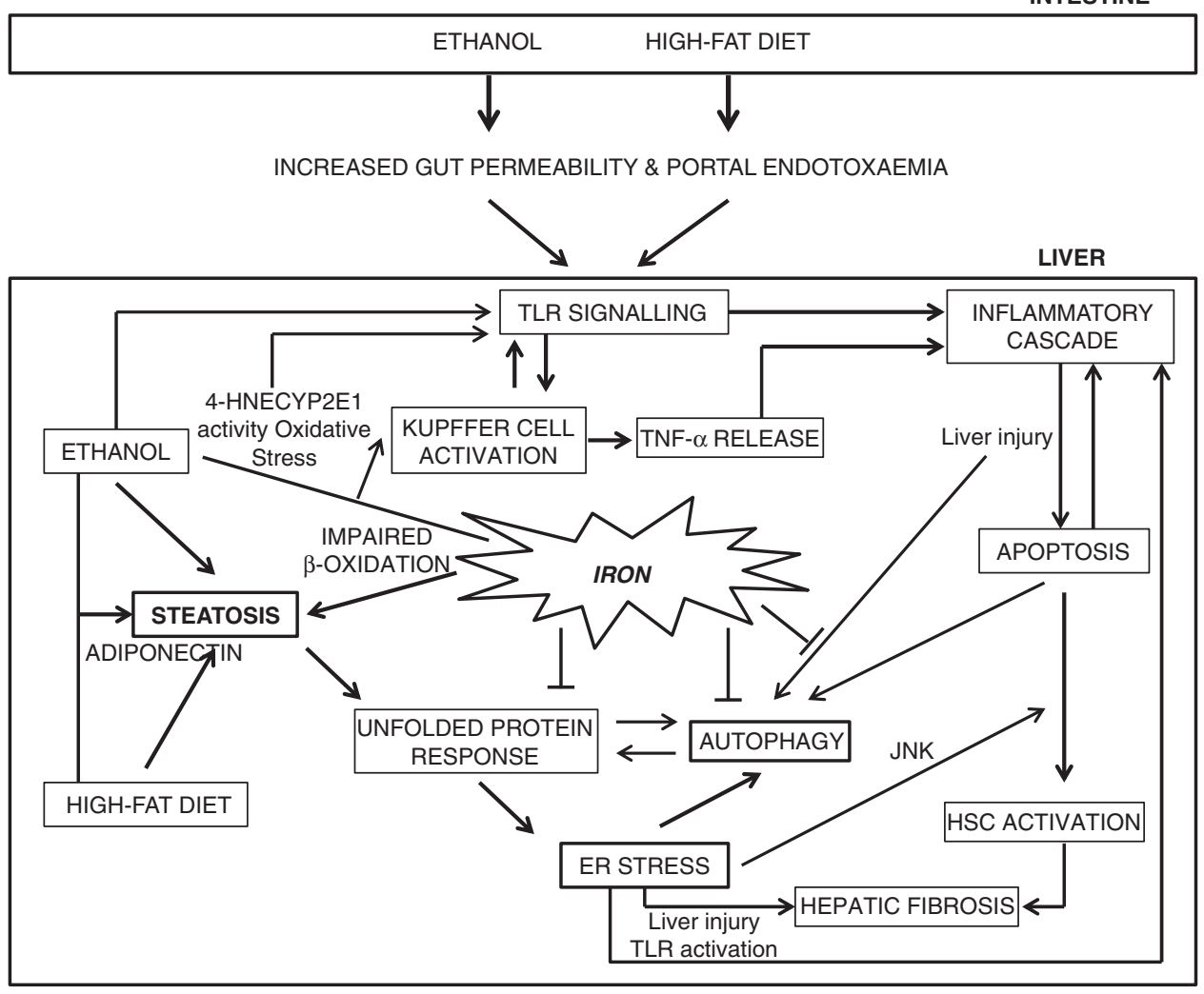

Figure 12 Pathways of interaction between iron, ethanol, and high-fat diet (HFD) in mediating chronic liver injury and fibrosis. Alcohol and HFD may increase intestinal permeability and increase hepatic TLR activation. This may lead to increased Kupffer cell activation and TNF- $\alpha$ release, triggering a proinflammatory cascade. This pathway may be primed by iron-induced oxidative stress. Both ethanol and HFD may lead to hepatic steatosis, a process accentuated by iron-induced impairment of mitochondrial $\beta$-oxidation. Hepatic steatosis leads to activation of the unfolded protein response, with potentially subsequent ER stress and modulation of autophagy. This process may be exacerbated by iron toxicity via regulation of the UPR and autophagic flux pathways. The increased ER stress may modulate apoptosis signaling pathways via JNK as well as promote cellular injury. Activation of TLR on HSC may lead to increased fibrogenic activity in the liver. ER, endoplasmic reticulum; HSC, hepatic stellate cells; TLR, Toll-like receptor.

NAFLD and ALD. ${ }^{12,13}$ Conversely, HFD and alcohol-induced steatosis may also suppress hepatocyte autophagy. ${ }^{38,39}$ An underlying mechanism for this observed iron effect remains unclear, but may relate to a synergistic effect on ethanolinduced reduction in $\mathrm{AMPK} \alpha$ activity in the liver via the mTOR pathway, or a decrease in fusion efficiency between autophagosomes and lysosomes because of diet-induced membrane lipid alterations. ${ }^{40,41}$ The role of iron in modulating autophagy is unclear, but iron-induced lyosomal damage and fragility could lead to impaired formation of the functioning of autophagosome function. ${ }^{6}$ Defective autophagy may also be an initiating factor for ER stress, given the central role of autophagy in the degradation of unfolded proteins and in the removal of superfluous ER membranes. ${ }^{42}$ The sequestosome protein p62 recognizes toxic protein aggregates and targets them for autophagic degradation. When autophagy is disrupted, an accumulation of p62 may lead to a deleterious activation of oxidative response genes. ${ }^{43}$ The reduced hepatic expression of key autophagy proteins, such as Atg3, Atg7, and beclin-1, in association with an accumulation of p62 in our mice with combined toxicity, supports the argument for an impaired autophagy response caused by iron overload, alcohol, and HFD. Furthermore, the expression of the microtubule protein LC3-I, a marker of autophagosome formation, and its conjugation product LC3-II, was decreased in the $\mathrm{Hfe}^{-/-}$as compared with the WT groups, indicating a link between iron and defective autophagy.

An interesting finding in this model is that of increased multiple TLR gene expression associated with hepatic iron overload following the ethanol-HFD co-feeding. TLRs are pattern recognition receptors that recognize pathogen-associated molecular patterns (PAMPs) and signal through adaptor molecules. ${ }^{44}$ Increased gut permeability secondary to increased alcohol and HFD intake can lead to increased levels of bacterial products such as lipopolysaccharide (LPS) in the portal circulation. ${ }^{45}$ This can lead to increased activation of Kupffer cells within the hepatic sinusoids. Current evidence suggests that increased TLR signaling is implicated in the development of alcoholic liver injury and NAFLD. ${ }^{46,47}$ The interaction between TLRs and PAMPs may lead to signaling through adaptor molecules and activation of the innate immune system involving TNF- $\alpha$. TLRs are expressed in 
hepatocytes, macrophages, as well as hepatic stellate cells, and their activation via TLR-dependent mechanisms may cause a cascade of inflammatory and stress-activated pathways as well as an enhancement of fibrogenesis. ${ }^{44}$ Ethanol and HFD ingestion are known to induce multiple TLRs in animal models. ${ }^{27}$ Mice deficient in TLR2 and TLR4 display attenuation in diet-induced hepatic steatosis and alcoholic liver injury. ${ }^{4-50}$ Although this study did not thoroughly examine the role of specific TLRs in detail, the increased expression of multiple TLRs in $\mathrm{Hfe}^{-/-}$mice following ethanol and HFD suggests a possible role of iron in modulating TLR signaling in liver diseases. The mechanisms behind this remain unclear, but may involve LPS-independent pathways as there is currently no evidence that Hfe deficiency or iron overload affects hepatic responses to portal endotoxemia. TLR activation is known to be associated with increased ER stress in the liver, and this finding may be consistent with the hypothesis that iron toxicity exacerbates alcohol-induced hepatic ER stress. ${ }^{51}$ An alternative explanation may be that increased TLR signaling reflects activation of hepatic stellate cells, which is primed for increased activity by iron toxicity following ethanol and HFD.

There were a number of surprising findings in our model of combined liver injury. First, the results reported above need to be interpreted in light of significantly elevated serum ethanol levels in the $\mathrm{Hfe}^{-1-}$ groups as compared with their WT counterparts. The reason for this is unclear in our study, but a possible explanation may lie with altered ethanol metabolism with iron overload or with Hfe protein dysfunction. A recent study has shown the importance of the $\beta$-catenin pathways in ethanol metabolism whereby $\beta$-catenin knockout mice had almost absent CYP2E1 induction after ethanol feeding followed by marked plasma ethanol levels. ${ }^{52}$ In the same vein, we postulate that iron excess may impair hepatic peroxisomal, mitochondrial, and microsomal function through oxidative stress injury pathways. Therefore, iron toxicity may lead to impaired alcohol and acetaldehyde dehydrogenase function as a result of oxidative injury to these organelles. This can be seen by the lack of CYP2E1 protein induction in the $\mathrm{Hfe}^{-/-}$mice following ethanol consumption as compared with the WT animals. This may in part explain the elevated serum ethanol levels that could further contribute to alcohol-induced hepatotoxicity in our model. Second, the lack of further increases in lipid peroxidation with combined ethanol, iron, and HFD despite increases in oxidative stress suggests that other oxidative stress-related pathways may play a role in disease modification related to this model. Lipid peroxidation has traditionally been viewed as a consequence of iron-induced oxidative stress, but the role of HFD in modulating tissue lipid peroxidation remains unclear and may provide an alternative explanation for the lack of an observed increase in MDA levels in the $\mathrm{Hfe}^{-/-}$mice. It is possible that ironinduced oxidative stress may lead to liver injury independent of the effects on lipid peroxidation, such as those pathways involved in cellular stress signaling.

In summary, we have shown that iron overload, even in mild excess, may enhance alcohol- and HFD-induced liver disease by modulating the cellular stress response pathways. The findings in study highlight the complex pathogenic interactions between iron, fat, and alcohol in the liver, many of them overlapping. The effects of each of these toxins are summarized in a flow diagram in Figure 12. This highlights the potentially crucial role of iron toxicity-mediated ER stress and impaired autophagy in the pathogenesis of liver fibrosis and may have future implications with regard to iron-chelating agents as antifibrotic therapies.

Supplementary Information accompanies the paper on the Laboratory Investigation website (http://www.laboratoryinvestigation.org)

\section{ACKNOWLEDGMENTS}

This research was supported by a project grant from the Queensland Health Smart Health Research Grant Scheme and by a PhD scholarship to TCHT from the Gallipoli Medical Research Foundation. VNS and GJA are recipients of Senior Research Fellowships from the National Health and Medical Research Council of Australia.

\section{DISCLOSURE/CONFLICT OF INTEREST}

The authors declare no conflict of interest.

1. Tilg $\mathrm{H}$, Diehl AM. Cytokines in alcoholic and nonalcoholic steatohepatitis. N Engl J Med 2000;343:1467-1476.

2. Nelson JE, Bhattacharya R, Lindor KD, et al. HFE C282Y mutations are associated with advanced hepatic fibrosis in Caucasians with nonalcoholic steatohepatitis. Hepatology 2007;46:723-729.

3. Nelson JE, Brunt EM, Kowdley KV. Lower serum hepcidin and greater parenchymal iron in nonalcoholic fatty liver disease patients with C282Y HFE mutations. Hepatology 2012;56:1730-1740.

4. Ganne-Carrie N, Christidis C, Chastang C, et al. Liver iron is predictive of death in alcoholic cirrhosis: a multivariate study of 229 consecutive patients with alcoholic and/or hepatitis $C$ virus cirrhosis: a prospective follow up study. Gut 2000;46:277-282.

5. Fletcher LM, Dixon JL, Purdie DM, et al. Excess alcohol greatly increases the prevalence of cirrhosis in hereditary hemochromatosis. Gastroenterology 2002;122:281-289.

6. Ramm GA, Ruddell RG. Hepatotoxicity of iron overload: mechanisms of iron-induced hepatic fibrogenesis. Semin Liver Dis 2005;25:433-449.

7. Tsukamoto $\mathrm{H}$, Horne $\mathrm{W}$, Kamimura $\mathrm{S}$, et al. Experimental liver cirrhosis induced by alcohol and iron. J Clin Invest 1995;96:620-630.

8. Silva $M$, Silva $M E$, de Paula $H$, et al. Iron overload alters glucose homeostasis, causes liver steatosis, and increases serum triacylglycerols in rats. Nutr Res 2008;28:391-398.

9. She H, Xiong S, Lin M, et al. Iron activates NF-kappaB in Kupffer cells. Am J Physiol Gastrointest Liver Physiol 2002;283:G719-G726.

10. Schroder M, Kaufman RJ. The mammalian unfolded protein response. Annu Rev Biochem 2005;74:739-789.

11. Wu J, Kaufman RJ. From acute ER stress to physiological roles of the Unfolded Protein Response. Cell Death Differ 2006;13:374-384.

12. Singh $\mathrm{R}$, Kaushik $\mathrm{S}$, Wang $\mathrm{Y}$, et al. Autophagy regulates lipid metabolism. Nature 2009;458:1131-1135.

13. Rautou PE, Mansouri A, Lebrec D, et al. Autophagy in liver diseases. J Hepatol 2010;53:1123-1134.

14. Dara L, Ji C, Kaplowitz N. The contribution of endoplasmic reticulum stress to liver diseases. Hepatology 2011;53:1752-1763. 
15. Xu J, Lai KK, Verlinsky A, et al. Synergistic steatohepatitis by moderate obesity and alcohol in mice despite increased adiponectin and p-AMPK. J Hepatol 2011;55:673-682.

16. Ji C. Mechanisms of alcohol-induced endoplasmic reticulum stress and organ injuries. Biochem Res Int 2012;2012:216450.

17. Ye D, Li FY, Lam KS, et al. Toll-like receptor-4 mediates obesity-induced non-alcoholic steatohepatitis through activation of X-box binding protein-1 in mice. Gut 2012;61:1058-1067.

18. Rautou PE, Mansouri A, Lebrec D, et al. Autophagy in liver diseases. J Hepatol 2010;53:1123-1134.

19. Tan TC, Crawford DH, Jaskowski LA, et al. Altered lipid metabolism in Hfe-knockout mice promotes severe NAFLD and early fibrosis. Am J Physiol Gastrointest Liver Physiol 2011;301:G865-G876.

20. Kleiner DE, Brunt EM, Van Natta M, et al. Design and validation of a histological scoring system for nonalcoholic fatty liver disease. Hepatology 2005;41:1313-1321.

21. Bligh EG, Dyer WJ. A rapid method of total lipid extraction and purification. Can J Biochem Physiol 1959;37:911-917.

22. Ohkawa H, Ohishi N, Yagi K. Assay for lipid peroxides in animal tissues by thiobarbituric acid reaction. Anal Biochem 1979;95:351-358.

23. Kirby DM, Thorburn DR, Turnbull DM, et al. Biochemical assays of respiratory chain complex activity. Methods Cell Biol 2007;80:93-119.

24. Stuart KA, Fletcher LM, Clouston AD, et al. Increased hepatic iron and cirrhosis: no evidence for an adverse effect on patient outcome following liver transplantation. Hepatology 2000;32:1200-1207.

25. Brunt EM, Olynyk JK, Britton RS, et al. Histological evaluation of iron in liver biopsies: relationship to HFE mutations. Am J Gastroenterol 2000;95:1788-1793.

26. Erridge C, Attina T, Spickett CM, et al. A high-fat meal induces lowgrade endotoxemia: evidence of a novel mechanism of postprandial inflammation. Am J Clin Nutr 2007;86:1286-1292.

27. Gustot T, Lemmers A, Moreno C, et al. Differential liver sensitization to toll-like receptor pathways in mice with alcoholic fatty liver. Hepatology 2006;43:989-1000.

28. Kouroku Y, Fujita E, Tanida I, et al. ER stress (PERK/elF2alpha phosphorylation) mediates the polyglutamine-induced LC3 conversion, an essential step for autophagy formation. Cell Death Differ 2007;14:230-239.

29. Corradini E, Pietrangelo A. Iron and steatohepatitis. J Gastroentero Hepatol 2012;27(Suppl 2):42-46.

30. Corpechot C, Barbu V, Wendum D, et al. Hypoxia-induced VEGF and collagen I expressions are associated with angiogenesis and fibrogenesis in experimental cirrhosis. Hepatology 2002;35: 1010-1021.

31. Hetz $C$. The unfolded protein response: controlling cell fate decisions under ER stress and beyond. Nat Rev Mol Cell Biol 2012;13:89-102.

32. Malhi H, Kaufman RJ. Endoplasmic reticulum stress in liver disease. J Hepatol 2011;54:795-809.

33. Schattenberg JM, Singh R, Wang Y, et al. JNK1 but not JNK2 promotes the development of steatohepatitis in mice. Hepatology 2006;43: 163-172.

34. Fuest $M$, Willim $K$, MacNelly $S$, et al. The transcription factor c-Jun protects against sustained hepatic endoplasmic reticulum stress thereby promoting hepatocyte survival. Hepatology 2012;55: 408-418.

35. Zhang K, Wang S, Malhotra J, et al. The unfolded protein response transducer IRE1alpha prevents ER stress-induced hepatic steatosis. EMBO J 2011;30:1357-1375.

36. Mizushima N, Levine B, Cuervo AM, et al. Autophagy fights disease through cellular self-digestion. Nature 2008;451:1069-1075.

37. Levine B, Kroemer G. Autophagy in the pathogenesis of disease. Cell 2008;132:27-42.

38. Czaja MJ. Functions of autophagy in hepatic and pancreatic physiology and disease. Gastroenterology 2011;140:1895-1908.

39. Amir M, Czaja MJ. Autophagy in nonalcoholic steatohepatitis. Expert Rev Gastroenterol Hepatol 2011;5:159-166.

40. You M, Matsumoto M, Pacold CM, et al. The role of AMP-activated protein kinase in the action of ethanol in the liver. Gastroenterology 2004;127:1798-1808.

41. Osna NA, Thomes PG, Jr TM. Involvement of autophagy in alcoholic liver injury and hepatitis C pathogenesis. World J Gastroenterol 2011;17:2507-2514.

42. Yang L, Li P, Fu S, et al. Defective hepatic autophagy in obesity promotes ER stress and causes insulin resistance. Cell Metab 2010;11:467-478.

43. Komatsu M, Waguri S, Koike M, et al. Homeostatic levels of p62 control cytoplasmic inclusion body formation in autophagy-deficient mice. Cell 2007:131:1149-1163.

44. Seki E, Brenner DA. Toll-like receptors and adaptor molecules in liver disease: update. Hepatology 2008;48:322-335.

45. Petrasek J, Mandrekar P, Szabo G. Toll-like receptors in the pathogenesis of alcoholic liver disease. Gastroenterol Res Pract 2010;2010:710381

46. Mathurin $P$, Deng QG, Keshavarzian A, et al. Exacerbation of alcoholic liver injury by enteral endotoxin in rats. Hepatology 2000;32: 1008-1017.

47. Ruiz AG, Casafont F, Crespo J, et al. Lipopolysaccharide-binding protein plasma levels and liver TNF-alpha gene expression in obese patients: evidence for the potential role of endotoxin in the pathogenesis of non-alcoholic steatohepatitis. Obes Surg 2007;17: 1374-1380.

48. Rivera CA, Gaskin L, Allman M, et al. Toll-like receptor-2 deficiency enhances non-alcoholic steatohepatitis. BMC Gastroenterol 2010;10:52.

49. Rivera CA, Adegboyega $\mathrm{P}$, van $\mathrm{RN}$, et al. Toll-like receptor-4 signaling and Kupffer cells play pivotal roles in the pathogenesis of nonalcoholic steatohepatitis. J Hepatol 2007;47:571-579.

50. Uesugi T, Froh M, Arteel GE, et al. Toll-like receptor 4 is involved in the mechanism of early alcohol-induced liver injury in mice. Hepatology 2001;34:101-108.

51. Martinon $\mathrm{F}$, Chen $\mathrm{X}$, Lee $\mathrm{AH}$, et al. TLR activation of the transcription factor XBP1 regulates innate immune responses in macrophages. Nat Immunol 2010;11:411-418.

52. Liu S, Yeh TH, Singh VP, et al. $\beta$-Catenin is essential for ethanol metabolism and protection against alcohol-mediated liver steatosis in mice. Hepatology 2012;55:931-940. 